\title{
Homeomorphisms of 3-manifolds and the realization of Nielsen number
}

\author{
BoJU JiAng ${ }^{1}$, Shicheng WANG ${ }^{1,2}$, AND YING-QING WU ${ }^{2}$
}

The Nielsen Conjecture for Homeomorphisms asserts that any homeomorphism $f$ of a closed manifold is isotopic to a homeomorphism realizing the Nielsen number of $f$, which is a lower bound for the number of fixed points among all maps homotopic to $f$. The main theorem of this paper proves this conjecture for all orientation preserving homeomorphisms on geometric or Haken 3-manifolds. It will also be shown that on many manifolds all orientation-preserving homeomorphisms are isotopic to fixed point free homeomorphisms.

The proof is based on the understanding of homeomorphisms on 2-orbifolds and 3-manifolds. Thurston's classification of surface homeomorphisms will be generalized to 2-dimensional orbifolds, which is used to study fiber preserving homeomorphisms of Seifert fiber spaces. Homeomorphisms on most Seifert fiber spaces are indeed isotopic to fiber preserving homeomorphisms, with the exception of four manifolds and orientation-reversing homeomorphisms on lens spaces or $S^{3}$. It will also be determined exactly which manifolds have a unique Seifert fibration up to isotopy. This information will be used to deform a homeomorphism to a certain standard map on each piece of the JSJ decomposition, as well as on the neighborhood of the decomposition tori, which will make it possible to shrink each fixed point class to a single point, and remove inessential fixed point classes.

\section{Introduction.}

Consider a map $f$ on a compact polyhedron $M$. Denote by Fix $(f)$ the set of fixed points of $f$. Two points $x, y \in \mathrm{Fix}(f)$ are equivalent (or Nielsen equivalent) if there is a path $\gamma$ from $x$ to $y$ such that $\gamma$ and $f \circ \gamma$ are rel $\partial \gamma$ homotopic. It can be shown that this is an equivalence relation, so it

\footnotetext{
${ }^{1}$ Partially supported by NSFC and MSTC grant.

${ }^{2}$ Research at MSRI supported in part by NSF grant \#DMS 9022140.
} 
divides Fix $(f)$ into equivalence classes, called the Nielsen classes. For each fixed point class one can define an index. A fixed point class is essential if its index is non-zero. The Nielsen number $N(f)$ of $f$ is then defined as the number of essential fixed point classes in Fix $(f)$. The important property of $N(f)$ is that it is a homotopy invariant of maps. In particular it gives a lower bound of the number of fixed points for all maps homotopic to $f$. One is referred to [J1] for an introduction to the Nielsen fixed point theory.

It is known that if $M$ is a compact manifold of dimension $\geq 3$, then $N(f)$ can be realized as the number of fixed points for some map homotopic to $f$, see $[\mathrm{Ki}, \mathrm{We}, \mathrm{Br}]$. This result is not true for continuous maps on hyperbolic surfaces [J2]. For homeomorphisms of manifolds, Nielsen conjectured that if $f$ is a surface homeomorphism then the Nielsen number is realized by the fixed point number of some homeomorphism isotopic to $f$. A proof of this conjecture was announced by Jiang [J3] and Ivanov [Iv], and given in detail by Jiang and Guo in [JG]. The following conjecture of Nielsen remains an open problem for manifolds of dimension 3 and 4.

The Nielsen Conjecture for Homeomorphisms. If $M$ is a closed manifold, and $f: M \rightarrow M$ is a homeomorphism, then $f$ is isotopic to a homeomorphism $g$ whose fixed point number equals $N(f)$.

This is trivial for 1-manifolds, and the work of Jiang and Guo [JG] proved it for 2-manifolds, which uses Thurston's classification of surface homeomorphisms. Kelly [Ke] gave a proof of this conjecture for homeomorphisms on manifolds of dimension at least 5 , using the fact that any null-homotopic simple closed curve in such a manifold bounds an embedded disc.

The main result of this paper is to prove this conjecture for orientationpreserving homeomorphisms on closed orientable 3-dimensional manifolds which are either Haken or geometric. Thurston's Geometrisation Conjecture asserts that these include all irreducible, orientable, closed 3-manifolds.

Theorem 9.1. Suppose $M$ is a closed orientable 3-manifold which is either Haken or geometric, and $f: M \rightarrow M$ is an orientation-preserving homeomorphism. Then $f$ is isotopic to a homeomorphism $g$ with \#Fix $(g)=$ $N(f)$.

There are eight geometries for 3-manifolds, see [Sc]. A Haken manifold can be decomposed along a canonical (possibly empty) set of tori into pieces, each of which is either Seifert fibred, or is a non-Seifert fibred atoroidal manifold. This decomposition is called the Jaco-Shalen-Johannson torus decomposition, or simply the JSJ decomposition. Thurston [Th2] proved 
that each component of the decomposed manifold admits a geometric structure. We will show that the homeomorphism on each individual piece $M_{i}$ is isotopic to a "standard homeomorphism", which has the property that no two components of the fixed point set of $\left.f\right|_{M_{i}}$ are equivalent on that piece, and the behavior of the homeomorphism near each fixed point component is well understood. Some major difficulties arise when we try to put the pieces together, because two fixed points being equivalent is a global property: Two components of the fixed point set of $\left.f\right|_{M_{i}}$ may be inequivalent in $M_{i}$, but equivalent in the whole manifold. See Remark 5.13 for an example. Therefore the homeomorphisms $\left.f\right|_{M_{i}}$ should not only satisfy the above conditions, but also some extra conditions to guarantee that after gluing the pieces together we still get a homeomorphism with good properties. This justifies the sophisticated definition of standard homeomorphisms given in Section 5. It can then be shown that if the restrictions of the homeomorphism on the pieces are standard homeomorphisms in that sense, then the global homeomorphism indeed has the property that each fixed point class is connected. The local behavior of the homeomorphism near each fixed point component will then enable us to shrink that component to a single point, and delete it if it is an inessential class.

For many manifolds, a much stronger result holds: All homeomorphisms on those manifolds are isotopic to fixed point free homeomorphisms. Define an orbifold $X(M)$ to be small if it is a sphere with at most three holes or cone points, or a projective plane with at most two holes or cone points; otherwise it is big.

Theorem 9.2. Let $M$ be a closed orientable 3-manifold which is either Haken or geometric. Then any orientation-preserving homeomorphism $f$ on $M$ is isotopic to a fixed point free homeomorphism, unless some component of the JSJ decomposition of $M$ is a Seifert fiber space with big orbifold.

In particular, the theorem applies to the following manifolds: (1) all Seifert fiber spaces whose orbifold is a sphere with at most 3 cone points or a projective plane with at most two cone points. This covers all manifolds admitting a $S^{3}$ or $S^{2} \times \mathbb{R}$ geometry, and four of the six Euclidean manifolds. (2) All manifolds obtained by gluing hyperbolic manifolds together along boundary tori. This includes all closed hyperbolic manifolds. (3) All Sol manifolds.

Our proof strongly depends on the knowledge of homeomorphisms on geometric manifolds, as well as homeomorphisms on 2-dimensional orbifolds. In Section 1 we will generalize Thurston's classification theorem of hyper- 
bolic surface homeomorphisms to homeomorphisms of hyperbolic orbifolds. We define periodic, reducible and pseudo-Anosov homeomorphisms on hyperbolic orbifolds $X$ in a natural way, in the sense that if $F$ is a surface covering of $X$, and $\tilde{f}$ is a lifting of a homeomorphism $f$ on $X$, then $f$ is pseudo-Anosov if and only if $\tilde{f}$ is.

Theorem 1.4. Suppose $X$ is a hyperbolic orbifold. Then any homeomorphism $f: X \rightarrow X$ is isotopic to either a periodic map, a reducible map, or a pseudo-Anosov map.

A similar result is proved in Section 2 for homeomorphisms on Euclidean orbifolds. These results are crucial to our proof because the orbifold of a Seifert fiber space $M$ has a natural orbifold structure, and any fiberpreserving homeomorphism on $M$ induces a homeomorphism of the orbifold.

In Section 3 we study the problem of which manifolds have a Seifert fibration such that every orientation-preserving homeomorphism is isotopic to a fiber-preserving one. This property is known to be true for all Seifert fiber spaces which are not covered by $S^{2} \times S^{1}, S^{3}$ or $T^{3}$ [Sc, Theorem 3.9], as well as for those manifolds which have a sphere orbifold with cone points of order $(2,3, p), p \geq 5$ or $(3,3, k), k \geq 2$ [BO, Proposition 3.1]. In Section 3 we will show that this is true for all but four Seifert fiber spaces.

Theorem 3.11. Suppose $M$ is a compact orientable Seifert fiber space which is not $T^{3}, M_{P(2,2)}, S^{1} \times D^{2}$, or $T \times I$. Then there is a Seifert fibration $p: M \rightarrow X(M)$, so that any orientation-preserving homeomorphism on $M$ is isotopic to a fiber-preserving homeomorphism with respect to this fibration. Moreover, if $M$ is not a lens space or $S^{3}$, then the result is true for all homeomorphisms on $M$.

A strong version of the converse is true: Not only does none of the four manifolds listed in the theorem have a "universal" invariant Seifert fibration, but also there exist homeomorphisms on these manifolds that do not preserve any Seifert fibration. As a corollary, we also completely determine all Seifert fiber spaces which have a unique Seifert fibration up to isotopy, see Corollary 3.12 for more details.

Two of the exceptional manifolds in Theorem 3.11 are closed. We need to find certain representatives for homeomorphisms on these manifolds separately. The manifold $T^{3}$ is easy: Each homeomorphism on $T^{3}$ is isotopic to a linear map. The manifold $M_{P(2,2)}$ is a Euclidean manifold with orbifold a projective plane with two cones of order 2 . This manifold will be treated 
in Section 4. It is shown that any homeomorphism on it is isotopic to an isometry with respect to a certain Euclidean metric.

Section 5 gives the definition of some basic concepts from fixed-point theory, including Nielsen class, index, and the Nielsen number of a map. We will also define two types of standard homeomorphisms on 3-manifolds, and prove some useful lemmas.

The remaining part of the paper uses the results in the earlier sections to give a proof of Theorem 9.1. Given a generic 3-manifold, the Jaco-ShalenJohannson decomposition cuts $M$ into hyperbolic and Seifert fibered pieces. Using Theorem 1.4, one can further cut the Seifert fibered components into pieces so that on each piece $M_{i}$ the homeomorphism $f$ induces an orbifold homeomorphism which is either periodic or pseudo-Anosov. The main result of Section 6 is to show that if $f$ is such a homeomorphism on a piece $M_{i}$, then it can be isotopic to a standard homeomorphism.

In Section 7, we will study torus fiber-preserving homeomorphisms on torus bundles over 1-orbifolds. These include twisted $I$-bundles over the Klein bottle, the union of two such manifolds along their boundary, $T \times I$, and torus bundles over $S^{1}$. An important lemma is that if $f$ is already standard on the boundary of a $T \times I$, then it is isotopic rel $\partial(T \times I)$ to a standard homeomorphism. This is useful in putting standard homeomorphisms on the pieces together to form a global standard homeomorphism. Also, the study of homeomorphisms on torus bundles over $S^{1}$ proves Theorem 9.1 for Sol manifolds. The theorem is proved in Section 8 for five other types of geometric manifolds; one is the hyperbolic manifolds, and the other four types are Seifert manifolds with spherical or Euclidean orbifolds.

The proof of Theorem 9.1 is completed in Section 9. With the previous results we can show that the homeomorphism is isotopic to one which is standard on each piece, including the collars of the decomposing tori. It will then be shown that in this case the global homeomorphism will have the property that each fixed point class is connected. The behavior of the homeomorphism in a neighborhood of each fixed point component is well understood, which enables us to shrink each fixed point class to a single point, and remove it if it is inessential. Theorem 9.2 is also proved in this section.

We refer the reader to $[\mathrm{Sc}]$ for basic definitions and properties of the eight 3 -manifold geometries, which is crucial in understanding this paper. Basic concepts about 3-manifold topology can be found in [Ja, He], in particular the Jaco-Shalen-Johannson decomposition of Haken manifolds and Seifert fiber spaces. See [Th1, Th2] for discussions on hyperbolic 3-manifolds.

We would like to thank Peter Scott for some helpful conversations about 
homeomorphisms on Seifert fiber spaces. Thanks also to the referee for his careful reading and many helpful comments; in particular, he brought the references [CV] and [BZ] to our attention, which eliminates some tedious arguments in proving the results of Section 4 in an earlier version of this paper.

Notations and Conventions. Given a set $X$ in $M$, we use $N(X)$ to denote a regular neighborhood of $X$, use $|X|$ to denote the number of components in $X$, and use $\# X$ to denote the number of points in $X$.

If $f: M \rightarrow M$ is a map, we use Fix $(f)$ to denote the set of fixed points of $f$, and use $N(f)$ to denote the Nielsen number of $f$.

A map $f$ on a Seifert fiber space $M$ is a fiber-preserving map if it maps each fiber to another fiber. Identifying each fiber of $M$ to a point, we get a set $X(M)$, which has a natural 2-dimensional orbifold structure [Sc, §3]. If $f$ is fiber-preserving, it induces a map $\widehat{f}: X(M) \rightarrow X(M)$. An isotopy $h_{t}(t \in I)$ of a Seifert fiber space is fiber-preserving if each $h_{t}$ is a fiberpreserving map. It is a fiberwise isotopy if $h_{t}$ maps each fiber to itself.

\section{2-orbifolds.}

\section{Classification of homeomorphisms on hyperbolic}

We refer the reader to [Sc, §2] for definitions about 2-dimensional orbifolds, their Euler characteristics, and other basic concepts and properties. All orbifolds in this paper are assumed compact and of dimension 2. Recall that an orbifold is good if it is covered by surface. Scott [Sc, Theorem 2.3] gave a list of all bad orbifolds without boundary. It is in fact the list of all bad orbifolds: for any orbifold $X$ with nonempty boundary, its double $\widehat{X}$ along $\partial X$ is clearly not in that list, thus covered by some surface, hence so is $X$ itself. An orbifold is hyperbolic if it has negative Euler characteristic. A hyperbolic orbifold is covered by a hyperbolic surface, and admits a hyperbolic structure with totally geodesic boundary. A reflector circle or reflector arc will be called a reflector. Denote by $S(X)$ the set of singular points of $X$. Thus each component of $S(X)$ is either a cone point, a reflector, or a union of reflector arcs joined at reflector corners.

Definition 1.1. A homeomorphism of an orbifold $X$ is a map $f: X \rightarrow X$ such that (1) $f$ is a homeomorphism of the underlying topological space, and (2) $f$ preserves the orbifold structure, i.e., it maps a cone point (resp. reflector corner) to a cone point (resp. reflector corner) of the same angle, and it maps a reflector to a reflector. 
Similarly, an isotopy on $X$ is a continuous family of orbifold homeomorphisms $f_{t}, 0 \leq t \leq 1$. In this case $f_{t}$ is called an isotopy from $f_{0}$ to $f_{1}$.

Definition 1.2. A set of mutually disjoint curves $C=\cup C_{i}$ in the interior of a hyperbolic orbifold $X$ is an admissible set if

(1) Each $C_{i}$ is either a circle disjoint from $S(X)$, or is a reflector circle, or is an arc with interior disjoint from $S(X)$ and with ends on cone points of order 2 .

(2) Each component of $X-\operatorname{Int} N(C)$ is a hyperbolic orbifold.

It is easy to see that if $C$ is an admissible set of $X$, then its lifting $\widetilde{C}$ on a compact covering surface $\widetilde{X}$ is an admissible set of $\widetilde{X}$ in the usual sense [FLP], i.e., it consists of mutually disjoint, mutually non-parallel, essential simple closed curves on $\widetilde{X}$.

Definition 1.3. Suppose $f: X \rightarrow X$ is a homeomorphism on a hyperbolic orbifold.

(1) $f$ is periodic if $f^{n}=i d$ for some $n$.

(2) $f$ is reducible if $f(C)=C$ for some admissible set $C$. The set $C$ is called a reducing set of $f$.

(3) $f$ is a pseudo-Anosov map if $S(X)$ has only cone points, and $f: X^{\prime} \rightarrow$ $X^{\prime}$ is a pseudo-Anosov map in the sense of Thurston, where $X^{\prime}$ is the punctured surface $X-S(X)$.

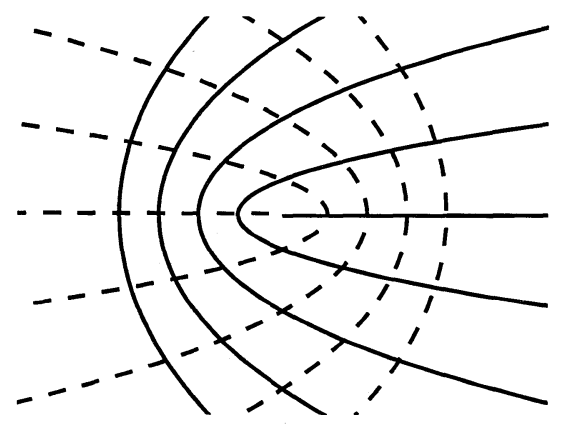

Figure 1.1. 
A pseudo-Anosov map has two transverse measured foliations, $\left(\mathcal{F}_{h}, \tau_{h}\right)$ and $\left(\mathcal{F}_{v}, \tau_{v}\right)$. A measured foliation on the orbifold $X$ is also a measured foliation of the underlying surface, except that a cone point of $X$ may be a singular point of the foliations with prong number 1, see Figure 1.1.

Thurston's classification of surface homeomorphisms [Th3] says that any homeomorphism on a hyperbolic surface is isotopic to either a periodic map, a reducible map, or a pseudo-Anosov map. For a proof of this theorem on orientable surfaces, see [FLP, Be, CB]. When the surfaces are nonorientable, this is proved in $[\mathrm{Wu}]$. The following theorem generalizes this theorem to homeomorphisms on hyperbolic orbifolds.

Theorem 1.4. Suppose $X$ is a hyperbolic orbifold. Then any homeomorphism $f: X \rightarrow X$ is isotopic to either a periodic map, a reducible map, or a pseudo-Anosov map.

Proof. If $X$ has reflector circles, let $C$ be the union of such. Then $f(C)=C$, and $X-\operatorname{Int} N(C)$ has the same Euler characteristic as that of $X$, so $f$ is a reducible map.

If $X$ has reflector arcs but no reflector circles, let $F$ be the underlying surface of $X$, and let $C^{\prime}=C_{1}^{\prime} \cup \ldots \cup C_{n}^{\prime}$ be the components of $\partial F$ containing some reflector arcs. Clearly we have $f\left(C^{\prime}\right)=C^{\prime}$. The set $C^{\prime}$ is isotopic to a set of curves $C=C_{1} \cup \ldots \cup C_{n}$ in the interior of $F$. By an isotopy we may assume that $f(C)=C$. The set $C$ cuts $X$ into $X_{1} \cup \ldots \cup X_{n} \cup Y$, where $X_{i}$ is an annulus between $C_{i}^{\prime}$ and $C_{i}$, and $Y$ is the remaining component. Since each $X_{i}$ is an annulus containing some reflector arcs, it is a hyperbolic orbifold. If $Y$ is also hyperbolic, then $C$ is an admissible set and we are done. So assume that $Y$ has nonnegative Euler characteristic. There are several cases.

Case 1: $Y$ is a Möbius band or an annulus with $\partial Y=C$. Replace $C$ by the central curve of $Y$.

Case 2: $Y$ is a disk with two cone points of order 2 and no other cone points. Replace $C=\partial Y$ by an arc in $\mathrm{Y}$ connecting the two cone points.

Case 3: $Y$ is either an annulus with one boundary on $\partial X$, or a disk with at most one cone point. Then $f$ is isotopic to a periodic map.

In all 3 cases $f$ is isotopic to a periodic map or a reducible map. Therefore the theorem holds if $X$ has some reflector arcs. 
Now assume that $X$ has no reflectors. Let $X^{\prime}=X-S(X)$, and let $f^{\prime}$ be the restriction of $f$ to $X^{\prime}$. If $f^{\prime}$ is isotopic to a periodic or pseudoAnosov map, then so is $f$, and we are done. So assume $f^{\prime}$ is isotopic to a reducible map $g^{\prime}$ with reducing curves $C^{\prime}$. Let $g$ be the corresponding homeomorphism on $X$. Then $g$ is isotopic to $f$ via an orbifold isotopy. If each component of $X-\operatorname{Int} N\left(C^{\prime}\right)$ is a hyperbolic orbifold, then $g$ is a reducible map and we are done. If some component $X_{i}$ of $X-\operatorname{Int} N\left(C^{\prime}\right)$ is not hyperbolic, it must be a disk $D$ with two cone points of order 2 . Replace $\partial D$ with an arc in $D$ connecting the two cone points. After doing this for all such $D$, we get a set $C$. By an isotopy in $D$ we may assume that $g(C)=C$. The components of $X-\operatorname{Int} N(C)$ are homeomorphic to the hyperbolic components of $X-\operatorname{Int} N\left(C^{\prime}\right)$. Therefore the modified $g$ is a reducible map on $X$ with $C$ the reducing set.

Theorem 1.5. Let $\tilde{X}$ be a compact covering surface of a hyperbolic orbifold $X$. If $f: X \rightarrow X$ is a pseudo-Anosov map and $\widetilde{f}: \widetilde{X} \rightarrow \widetilde{X}$ is a lifting of $f$, then $\widetilde{f}$ is a pseudo-Anosov map in the sense of Thurston.

Proof. Let $Y$ be the underlying surface of $X$. Then $f$ defines a generalized pseudo-Anosov map on $Y$, in the sense that the cone points might be singular points of the foliations with prong number $p=1$. Since $\widetilde{X}$ is a surface, if $y$ is a point in $\widetilde{X}$ which covers a cone point of angle $2 \pi / k$, then the foliations have prong number $k p \geq 2$ at $y$. It follows that the liftings of the foliations are foliations with no prong number 1 singularities in the interior of $\widetilde{X}$, and are preserved by $\widetilde{f}$. Therefore $\widetilde{f}$ is a pseudo-Anosov map in the sense of Thurston (see [FLP]).

Suppose that $S$ is a hyperbolic surface. Let $\Lambda$ be a finite group acting on $S$. Then $X=S / \Lambda$ is a hyperbolic orbifold. If $f: S \rightarrow S$ is a $\Lambda$-equivariant map, then it induces a map $f^{\prime}: X \rightarrow X$. Thus the theory of equivariant maps on surfaces follows from that on orbifolds. To be more precise, we make the following definitions.

Definition 1.6. Let $\Lambda$ be a finite group acting on a hyperbolic surface $S$.

(1) A homeomorphism $f: S \rightarrow S$ is a $\Lambda$-homeomorphism if $f \circ \lambda=\lambda \circ f$ for all $\lambda \in \Lambda$.

(2) An isotopy $f_{t}, 0 \leq t \leq 1$, is a $\Lambda$-isotopy if $f_{t}$ is a $\Lambda$-homeomorphism for all $t$. 
(3) A $\Lambda$-homeomorphism $f: S \rightarrow S$ is a $\Lambda$-reducible map if there is an admissible set $C=C_{1} \cup \ldots \cup C_{n}$ such that $f(C)=C$, and $\lambda(C)=C$ for all $\lambda \in \Lambda$.

(4) A $\Lambda$-homeomorphism $f: S \rightarrow S$ is a $\Lambda$-pseudo-Anosov map if $f$ is a pseudo-Anosov map in the sense of Thurston, and the measured foliations are $\Lambda$-invariant.

Since any $\Lambda$-homeomorphism $f$ on $S$ induces a homeomorphism $f^{\prime}$ on the orbifold, and an isotopy of $f^{\prime}$ induces a $\Lambda$-isotopy of $f$, the following $\Lambda$ equivariant version of Thurston classification theorem is a direct consequence of Theorem 1.4 .

Corollary 1.7. If $f: S \rightarrow S$ is a $\Lambda$-homeomorphism, then it is $\Lambda$-isotopic to a $\Lambda$-homeomorphism $g$ which is either a periodic $\Lambda$-homeomorphism, a $\Lambda$-reducible map, or a $\Lambda$-pseudo-Anosov map.

In [Be] Bers proved Thurston's classification theorem using quasiconformal mapping theory. Corollary 1.7 is also true in this category. A conformal structure $\sigma$ on $S$ is $\Lambda$-invariant if all maps in $\Lambda$ are conformal maps with respect to $\sigma$. Similarly, a quadratic differential $\omega$ is $\Lambda$-invariant if

$$
\lambda^{*}(\omega)=\omega \quad \text { or } \quad \lambda^{*}(\omega)=\bar{\omega}
$$

depending on whether $\lambda$ is orientation-preserving or orientation-reversing. A $\Lambda$-homeomorphism $f$ is a $\Lambda$-pseudo-Anosov map in the sense of Bers if there is a $\Lambda$-invariant conformal structure $\sigma$ on $S$, such that $f$ is an extremal map as defined in [Be], and its initial and terminal differential $\omega$ is $\Lambda$-invariant. Notice that such a differential defines a pair of $\Lambda$-invariant transverse measured foliations, preserved by the homeomorphism $f$. Hence such a homeomorphism is a $\Lambda$-pseudo-Anosov map as defined before. The proof of $[\mathrm{Wu}]$ can be modified to prove Corollary 1.7 in the conformal setting.

Definition 1.8. A reducing set $C$ of $f$ is called a complete reducing set if $f$ is isotopic to a homeomorphism $g$ with $g(N(C))=N(C)$, and $g^{n_{i}}$ restricted to $X_{i}$ is either a periodic map or a pseudo-Anosov map, where $X_{i}$ is an arbitrary component of $X-\operatorname{Int} N(C)$, and $n_{i}$ is the least positive integer such that $g^{n_{i}}$ maps $X_{i}$ to itself.

Remark 1.9. Clearly a maximal reducing set of $f$ is a complete reducing set. A minimal complete reducing set is called a canonical reducing set. It can be shown that if $C$ is a canonical reducing set of $f$, then its lifting $\widetilde{C}$ 
is a canonical reducing set of a lifting of $f$ on a compact covering surface $\widetilde{X}$ of $X$. Since the canonical reducing set of a surface homeomorphism is unique [Wu, Theorem 1], it follows that the canonical reducing set for $f$ is also unique, and it is empty if and only if $f$ is isotopic to a periodic or pseudo-Anosov map.

We now consider a fiber-preserving homeomorphism $f$ on an orientable Seifert fiber space $M$ which has hyperbolic orbifold $X(M)$. Let $p: M \rightarrow$ $X(M)$ be the projection map. Let $\widehat{f}: X(M) \rightarrow X(M)$ be the orbifold homeomorphism induced by $f$. Recall that a torus $T$ in $M$ is a vertical torus if it is a union of fibers in $M$.

Lemma 1.10. There is a collection of vertical tori $\mathcal{T}$ in $M$, and a homeomorphism $g$ which is isotopic to $f$ via a fiber-preserving isotopy, such that

(1) $g(N(\mathcal{T}))=N(\mathcal{T})$;

(2) The orbifold of each component of $M-\operatorname{Int} N(\mathcal{T})$ is either hyperbolic or a disk with two cone points of order 2 ; and

(3) If $M_{i}$ is a component of $M-\operatorname{Int} N(\mathcal{T})$ with hyperbolic orbifold, and $g\left(M_{i}\right)=M_{i}$, then the orbifold map $\widehat{g}: X\left(M_{i}\right) \rightarrow X\left(M_{i}\right)$ is either periodic or pseudo-Anosov.

Proof. Recall that since $X(M)$ is the orbifold of an orientable Seifert fiber space, it has no reflectors because reflectors correspond to solid Klein bottles [Sc, p. 430]. Choose a complete reducing set $C$ on $X(M)$ for the homeomorphism $\widehat{f}$, and modify it as follows: If $\alpha$ is an arc in $C$ connecting two cone points of order 2 , replace it by the curve $\alpha^{\prime}=\partial N(\alpha)$. We can modify $f$ via a fiber-preserving isotopy, to get a homeomorphism $g$ such that $\widehat{g}(N(C))=N(C)$, and the $\mathrm{r}$ estriction of $\widehat{g}$ to each hyperbolic component of $X(M)-\operatorname{Int} N(C)$ is either periodic or pseudo-Anosov. It is now clear that the homeomorphism $g$ and the collection of tori $\mathcal{T}=p^{-1}(C)$ satisfy the conclusion of the lemma.

\section{Classification of homeomorphisms on Euclidean orbifolds.}

A euclidean orbifold is an orbifold $X$ such that its orbifold Euler number is zero. A curve $C$ in $X-S(X)$ is an essential curve of $X$ if no component of $X-\operatorname{Int} N(C)$ is a disk with at most one cone point, or an annulus containing no singular points of $X$ and having one boundary on $\partial X$. Recall that a 
homeomorphism $f$ on a torus is an Anosov map if there is a pair of transverse measured foliations $\left(\mathcal{F}_{1}, u_{1}\right)$ and $\left(\mathcal{F}_{2}, u_{2}\right)$ such that $f$ preserves the foliations, and the measures $u_{i}$ are changed by $f\left(u_{1}\right)=\lambda u_{1}$, and $f\left(u_{2}\right)=(1 / \lambda) u_{2}$, where $\lambda>1$. We do not define Anosov maps on other euclidean surfaces: as we will see, all homeomorphisms on them are isotopic to periodic ones.

Definition 2.1. Let $f: X \rightarrow X$ be an orbifold homeomorphism on a euclidean orbifold $X$.

(1) $f$ is periodic if $f^{n}=i d$ for some $n$.

(2) $f$ is reducible if $f(C)=C$ for some essential curve $C$ of $X$. We call $C$ a reducing curve of $f$.

(3) If $S(X) \neq \emptyset$, then $f: X \rightarrow X$ is an Anosov map if $f: X^{\prime} \rightarrow X^{\prime}$ is a pseudo-Anosov map in the sense of Thurston, where $X^{\prime}=X-S(X)$.

Lemma 2.2 ([Li, Lemma 5]). Any homeomorphism $f: K \rightarrow K$ on a Klein bottle is isotopic to either the identity map or an involution.

Proof. Lickorish [Li, Lemma 5] proved that $\mathcal{H}(K) \cong \mathbb{Z}_{2} \times \mathbb{Z}_{2}$, and gave the representatives of the isotopy classes. It is easily seen that each isotopy class contains an involution.

If $F$ is a surface, we use $F\left(p_{1}, \ldots, p_{k}\right)$ to denote an orbifold with underlying surface $F$, and with cone points of order $p_{1}, \ldots, p_{k}$. If we use $S, P, T, K, D, A, U$ to denote the sphere, projective plane, torus, Klein bottle, disk, annulus and Möbius band, respectively, then a euclidean orbifold without reflector is one of the following:
(1) $T, K, A$, or $U$;
(2) $D(2,2)$;
(3) $P(2,2)$;
(4) $S(2,3,6), S(2,4,4), S(3,3,3)$;
(5) $S(2,2,2,2)$.

Lemma 2.3. If $f: X \rightarrow X$ is a homeomorphism on $X=P(r, s)$, then $f$ is isotopic to a periodic map.

Proof. Consider $P(r, s)$ as $D(r, s) / \rho$, where $\rho$ is the antipodal map on $\partial D$. Let $\alpha$ be an arc in $P(r, s)$ connecting the two cone points $a, b$, having minimal intersection number with $\partial D / \rho$ in its isotopy class. Thus on $D(r, s)$ 
$\alpha$ consists of several $\operatorname{arcs} \alpha_{0}, \ldots, \alpha_{k}$. We claim that $k \leq 1$. Assume $k \geq 2$. Denote by $\alpha_{i}(1)$ and $\alpha_{i}(0)$ the head and tail of $\alpha_{i}$, respectively. Let $\beta_{+}, \beta_{-}$ be the two components of $\partial D-\left\{\alpha_{0}(1), \alpha_{k}(0)\right\}$. The minimality of $k$ means that each $\alpha_{i}(i \neq 0, k)$ is an essential arc on $D(r, s)-\alpha_{0} \cup \alpha_{k}$, so it has one endpoint on each of $\beta_{+}, \beta_{-}$; hence each of $\beta_{+}, \beta_{-}$contains exactly $k-1$ endpoints of $\cup \alpha_{i}$. Since $\rho$ is an involution identifying the endpoints of $\cup \alpha_{i}$ on $\partial D$ to each other, we see that for any point $x$ on $\partial D$, the two arcs $\partial D-\{x, \rho(x)\}$ must contain the same number of points in $\cup \alpha_{i} \cap \partial D$. In particular, $\rho$ must identify $\alpha_{0}(1)$ to $\alpha_{k}(0)$, which implies that $\alpha$ has more than one component, contradicting the assumption that $\alpha$ is an arc.

Now suppose $\alpha, \alpha^{\prime}$ are two arcs connecting $a$ to $b$, which have the same minimal intersection number with $\partial D$. By the above, the intersection number is either 0 or 1 . It is clear that in this case these two arcs are isotopic rel $\partial$. Therefore up to isotopy there are exactly two arcs connecting $a$ to $b$, say $\alpha$ and $\beta$, which can be chosen to have disjoint interiors, so $\alpha \cup \beta$ is a circle. Since $f: X \rightarrow X$ is a homeomorphism, it either preserves or permutes these two isotopy classes, hence up to isotopy we may assume that $f(\alpha \cup \beta)=\alpha \cup \beta$, and $f$ is a periodic map on $N(\alpha \cup \beta)$. Since $D^{\prime}=X-\operatorname{Int} N(\alpha \cup \beta)$ is a disk with no cone points, $\left.f\right|_{D^{\prime}}$ is rel $\partial D^{\prime}$ isotopic to a periodic map.

Proposition 2.4. If a euclidean orbifold $X$ is not $T$ or $S(2,2,2,2)$, then any homeomorphism $f$ on $X$ is isotopic to a periodic one.

Proof. First assume that $X$ has no reflectors. The result is obvious when $X=A$ or $U$, and has been proved for $K$ and $P(2,2)$ in Lemmas 2.2 and 2.3 respectively. In all other cases $X-S(X)$ is a pair of pants, so any homeomorphism on $X-S(X)$ (hence on $X$ ) is periodic.

Now assume $X$ has no reflector circles but has some reflector arcs. By an isotopy we may assume that $f$ is already a periodic map on $N(C)$, where $C$ is the union of $\partial X$ and the reflectors. Since $X$ has no reflector circles but some reflector arcs, the Euler characteristic of $N(C)$ is negative, no matter whether it has reflector corners or not. Thus $X-\operatorname{Int} N(C)$ is an orbifold with nonempty boundary, which has positive Euler number but no reflectors, hence it must be a disk with at most one cone point. It follows that $f$ is rel $N(C)$ isotopic to a periodic map.

If $X$ has some reflector circles, one can ignore these reflector circles to get another orbifold $X^{\prime}$. Clearly $f: X \rightarrow X$ is isotopic to a periodic map if and only if $f: X^{\prime} \rightarrow X^{\prime}$ is, so the result follows from the above two cases. 
Theorem 2.5. (a) A homeomorphism $f$ on a euclidean orbifold $X$ is isotopic to either a periodic map, a reducible map, or an Anosov map.

(b) Let $\tilde{X}$ be a surface that covers $X$, and let $\tilde{f}: \widetilde{X} \rightarrow \widetilde{X}$ be a lifting of $f$. If $f$ is periodic (resp. reducible, Anosov) then $\widetilde{f}$ is also periodic (resp. reducible, Anosov).

Proof. (a) If $X=T$, this is a well known classical result. If $X=S(2,2,2,2)$, then $X-S(X)$ is a hyperbolic surface, so the result follows immediately from the definition above and Thurston's classification theorem. In all other cases by Proposition 2.4 the homeomorphism $f$ is isotopic to a periodic map.

(b) This is quite obvious if $f$ is periodic or reducible, or if $X=T$. Suppose $X=S(2,2,2,2), \widetilde{X}=T$, and $f$ is Anosov. By the proof of Theorem 1.5 , the measured foliations on $X$ lift to measured foliations on $\widetilde{X}$ which have no prong number 1 singularities. Recall that if a foliation on a surface $F$ has singular points of prong number $p_{1}, \ldots, p_{k}$, then the Euler characteristic of $F$ is $\sum\left(2-p_{i}\right) / 2$. Since the Euler characteristic of $\widetilde{X}$ is zero, the lifted foliations on $\tilde{X}$ have no singularities. Thus by definition $\tilde{f}$ is Anosov.

Remark 2.6. Not all results of hyperbolic orbifolds generalize to euclidean orbifolds. For example, if $X$ is a hyperbolic orbifold, then two homeomorphisms on $X$ are isotopic if and only if their liftings are isotopic. However, there is a homeomorphism on $S(2,2,2,2)$ which permutes the singular points (so it is not isotopic to the identity map), and yet its lifting to the torus is isotopic to the identity map.

\section{Invariant Seifert fibrations for homeomorphisms.}

In this section we will study the problem of which manifolds $M$ have a Seifert fibration such that every orientation-preserving homeomorphism on $M$ is isotopic to a fiber-preserving homeomorphism with respect to this fibration. Theorems 3.1 and 3.2 are what we can find in the literature. The results in this section will deal with the remaining cases. It turns out that all but four Seifert fiber spaces have this property. Moreover, if the manifold is not a lens space or $S^{3}$, then the fibration is preserved by orientation-reversing homeomorphisms as well. See Theorem 3.11 below.

Theorem 3.1 ([Sc, Theorem 3.9]). Let $M, N$ be compact Seifert fiber spaces (with fixed Seifert fibrations), and let $f: M \rightarrow N$ be a homeomor- 
phism. Then $f$ is homotopic to a fiber preserving homeomorphism (and hence an isomorphism of Seifert bundles) unless one of the following occurs.

(a) $M$ is covered by $S^{3}$ or $S^{2} \times \mathbb{R}$,

(b) $M$ is covered by $T^{3}$,

(c) $M$ is $S^{1} \times D^{2}$ or a I-bundle over the torus or Klein bottle.

It is now known that two homeomorphisms of Seifert fiber spaces are homotopic if and only if they are isotopic, see [Wa] for Haken manifolds, [Sc2] for irreducible Seifert manifolds with infinite fundamental groups, and [BO, RB, HR, R, B, La] for various cases of Seifert manifolds covered by $S^{3}$ and $S^{2} \times S^{1}$. Thus the word "homotopic" in the theorem can be replaced by "isotopic". The theorem says that if $M$ is not one of the listed manifolds, then Seifert fibrations on $M$ are unique up to isotopy.

We will restrict our discussion on orientable manifolds. For $S^{1} \times D^{2}$ and $T \times I$, there are obviously homeomorphisms which are not isotopic to any fiber-preserving homeomorphisms with respect to any Seifert fibration. If $M$ is a twisted $I$-bundle over the Klein bottle, then $M$ has two Seifert fibrations up to isotopy [WW, Lemma 1.1]. Note that those two Seifert fibrations have different orbifolds. Let $p: M \rightarrow X(M)$ be one of the Seifert fibrations. Then $p \circ f: M \rightarrow X(M)$ is another Seifert fibration with the same orbifold. Since these two Seifert fibrations have the same orbifold, [WW, Lemma 1.1] implies that they are isotopic, that is, there is a homeomorphism $\varphi$ of $M$, isotopic to the identity, so that $f \circ \varphi$ is a fiber-preserving map. It follows that $f$ is isotopic to a fiber-preserving homeomorphism with respect to each of the two Seifert fibrations of $M$. It remains to consider the manifolds covered by $S^{3}, S^{2} \times \mathbb{R}$, or $T^{3}$.

Theorem 3.2 ([BO, Proposition 3.1]). Suppose $M$ is a Seifert manifold with orbifold $S(2,3, p), p \geq 5$ or $S(3,3, q), q \geq 2$. Then every homeomorphism $f$ of $M$ is isotopic to a fiber-preserving homeomorphism of $M$.

By [Ja, VI.17], Seifert fibration structures on the manifolds in Theorem 3.2 are unique up to isomorphism. Theorem 3.2 says that it is unique up to isotopy. In this section we will discuss the remaining cases. The following lemma is useful in detecting homeomorphisms which are isotopic to fiberpreserving homeomorphisms. Note that the lemma is not true if $X(M)$ is a $S(p, q)$ or $D(p)$, since there are many non isotopic fibrations on lens spaces and the solid torus, all having one fiber in common. 
Lemma 3.3. Let $p: M \rightarrow X(M)$ be a Seifert fibration of an orientable 3-manifold $M$, such that $X(M)$ is not a sphere with at most two cone points or a disk with at most one cone point. Let $f: M \rightarrow M$ be a homeomorphism. If there is a fiber $\alpha$ of $M$ such that $f(\alpha)$ is isotopic to another fiber, then $f$ is isotopic to a fiber preserving homeomorphism with respect to the fibration $p$.

Proof. By an isotopy we may assume that $f(\alpha)$ is a fiber of $M$, and $f$ maps a fibered neighborhood $N(\tau)$ to a fibered neighborhood $N(f(\alpha))$. Let $M^{\prime}=$ $M-\operatorname{Int} N(\alpha)$, and let $N^{\prime}=M-\operatorname{Int} N(f(\alpha))$, with Seifert fibrations inherited from that of $M$. These are manifolds with boundary, so by Theorem 3.1 the map $\left.f\right|_{M^{\prime}}$ is isotopic to a fiber-preserving map unless $M^{\prime}$ is one of the manifolds listed in Theorem 3.1(c). If $M^{\prime}$ is a solid torus or a $T \times I$, then $X(M)$ is either a sphere with at most two cone points or a disk with at most one cone point, which have been excluded in the lemma. The remaining case is that $M^{\prime}$ and $N^{\prime}$ are twisted $I$-bundles over the Klein bottle. Recall that up to isotopy there are only two Seifert fibrations on a twisted $I$-bundle over the Klein bottle, with orbifold $D(2,2)$ and the Möbius band respectively [WW, Lemma 1.1]. Now the orbifolds $X\left(M^{\prime}\right)$ and $X\left(N^{\prime}\right)$ are both obtained from $X(M)$ by removing a disk with possibly one cone point, so they are both orientable or both nonorientable. It follows that $\left.f\right|_{M^{\prime}}$ is isotopic to a fiber-preserving homeomorphism. Extending the isotopy over $M$, we get a homeomorphism $g: M \rightarrow M$, which is fiber-preserving on $M^{\prime}$. Now $\left.g\right|_{N(\alpha)}$ maps the fibered solid torus $N(\alpha)$ to a fibered solid torus $N(f(\alpha))$, sending a fiber on $\partial N(\alpha)$ to a fiber on $\partial N(f(\alpha))$. By [Ja, Lemma VI.19], $\left.g\right|_{N(\alpha)}$ is rel $\partial N(\alpha)$ isotopic to a fiber-preserving map.

Denote by $\mathcal{H}(M)$ the homeotopy group of $M$, i.e., the group of all homeomorphisms modulo the subgroup of all those isotopic to identity. Denote by $\mathcal{H}^{+}(M)$ the subgroup represented by orientation-preserving homeomorphisms. We do not consider $S^{3}$ or $S^{2} \times S^{1}$ as lens spaces.

Lemma 3.4. Any lens space $M$ has a Seifert fibration $p: M \rightarrow X(M)$, such that any orientation-preserving homeomorphism $f$ on $M$ is isotopic to a fiber preserving homeomorphism with respect to this fibration.

Proof. Hodgson and Rubinstein [HR, Theorem 5.6 and Corollary 5.7] proved that $\mathcal{H}(M) \cong \mathbb{Z}_{2}, \mathbb{Z}_{2}+\mathbb{Z}_{2}$, or $\mathbb{Z}_{4}$, and gave explicit representatives of the 
isotopy classes. The orientation-preserving ones clearly preserve the Seifert fibration induced by the Hopf fibration of $S^{3}$.

Lemma 3.5. If $p: M \rightarrow X(M)$ is a Seifert fibration with $X(M)=$ $S(2,2, k)$ for some $k>1$, then any homeomorphism $f: M \rightarrow M$ is isotopic to a fiber preserving homeomorphism with respect to this fibration.

Proof. Let $\alpha$ be an arc on $X(M)$ connecting two cone points of order 2 . Then $p^{-1}(\alpha)$ is a Klein bottle $K_{1}$ in $M$. Note that if $k=2$ then there are three cone points of order 2, so there are three such arcs, and hence three Klein bottles $K_{1}, K_{2}, K_{3}$. It was shown in the proof of [R, Theorem 6] that $f$ can be deformed by an isotopy so that if $k \neq 2$ then $f$ maps $K_{1}$ to itself, and if $k=2$ then $f$ maps $K_{1}$ to one of the $K_{i}$. Note that each $K_{i}$ is a union of fibers of $M$, so it gives a fibration of $K_{i}$ with orbifold an arc with two cone points. A regular fiber of $K_{i}$ is a separating essential simple closed curve, which is unique up to isotopy, hence $f$ can be deformed by a global isotopy, so that $f$ preserves the fibration on $K_{i}$. Since the regular fibers of $K_{i}$ are regular fibers of $M$, it follows from Lemma 3.3 that $f$ is isotopic to a fiber-preserving homeomorphism.

Lemma 3.6. Suppose $p: M \rightarrow X(M)$ is a Seifert fibration of a spherical manifold $M$ with orbifold $X(M)=S(2,3,4)$. Then any homeomorphism $f: M \rightarrow M$ is isotopic to a fiber-preserving homeomorphism with respect to this fibration.

Proof. Identify $X(M)$ with the unit sphere in $\mathbb{R}^{3}$ so that the three cone points lie on the $x y$-plane. Let

$$
\widehat{g}: X(M) \rightarrow X(M)
$$

be the reflection along the $x y$-plane. There is a fiber-preserving involution

$$
g: M \rightarrow M
$$

with $\widehat{g}$ as its orbifold homeomorphism, constructed as follows: Removing a small neighborhood $N\left(c_{i}\right)$ for each cone point $c_{i}$, we get a three-punctured sphere $P \subset X(M)$, and $p^{-1}(P)=P \times S^{1}$. Define

$$
g: P \times S^{1} \rightarrow P \times S^{1}
$$


by $g(x, y)=(\widehat{g}(x), \bar{y})$, where $\bar{y}$ is the complex conjugate of $y$. The restriction of $g$ on each boundary torus of $P \times S^{1}$ is an orientation-preserving involution, which preserves the slope of any curve. Hence it extends to a fiber preserving involution on each fibered solid torus $p^{-1}\left(N\left(c_{i}\right)\right)$, resulting the required homeomorphism $g$.

The manifold $P \times S^{1}$ has a product fibration. Its fiber and the boundary of $P$ set up a longitude-meridian pair $\left(l_{i}, m_{i}\right)$ for each boundary component $T_{i}$ of $P \times S^{1}$. Denote by $M\left(p_{1}, q_{1} ; p_{2}, q_{2} ; p_{3}, q_{3}\right)$ the manifold obtained by attaching a solid torus $V_{i}$ to each $T_{i}$, so that a meridian of $V_{i}$ is glued to the curve $p_{i} m_{i}+q_{i} l_{i}$ on $T_{i}$. The manifold $M$ can be written as $M\left(2,1 ; 3, b_{2} ; 4, b_{3}\right)$, where $b_{2}= \pm 1$. By reversing the orientation of $M$ if necessary, we may assume that $b_{2}=1$, so $M=M\left(2,1 ; 3,1 ; 4, b_{3}\right)$. The first homology group of $M$ has a presentation

$$
\begin{gathered}
H_{1}(M)=\left\langle m_{1}, m_{2}, m_{3}, l\right| m_{1}+m_{2}+m_{3}=0,2 m_{1}+l=0 \\
\left.3 m_{2}+l=0,4 m_{3}+b_{3} l=0\right\rangle .
\end{gathered}
$$

It follows that

$$
H_{1}(M)=\mathbb{Z}_{\left|12+6 b_{3}+8 b_{2}\right|}=\mathbb{Z}_{\left|20+6 b_{3}\right|},
$$

see also [Or, $\S 6.2$, Theorem 2]. Thus $H_{1}(M)=\mathbb{Z}_{2}$ if and only if $b_{3}=-3$. The homeomorphism $g$ defined above sends each generator to its negative. Hence if $H_{1}(M)=\mathbb{Z}_{p}$ and $p \neq 2$, then $g_{*}: H_{1}(M) \rightarrow H_{1}(M)$ is not the identity. It follows that $g$ is not isotopic to the identity map if $b_{3} \neq-3$.

According to Rubinstein and Birman [RB, Main Theorem], the homeotopy group $\mathcal{H}(M)$ of $M$ is either $\mathbb{Z}_{2}$ or a trivial group, and it is trivial if and only if $b_{3}=-3$. We have shown that when $b_{3} \neq-3$, the homeomorphism $g$ is a nontrivial element in $\mathcal{H}(M)$, hence any homeomorphism on $M$ is isotopic to either $g$ or the identity map. When $b_{3}=-3, \mathcal{H}(M)$ is trivial, so all homeomorphisms are isotopic to the identity map. Since both $g$ and the identity map are fiber preserving, the result follows.

Lemma 3.7. Let $\rho: M \rightarrow S^{1}$ be a torus bundle structure on $M$. Let $f: M \rightarrow M$ be a homeomorphism. Then either $M$ is an $S^{1}$ bundle over $T^{2}$, or $f$ is isotopic to a torus fiber preserving homeomorphism with respect to the fibration $\rho$.

Proof. Let $T$ be a torus fiber in $M$, and let $T^{\prime}=f(T)$. Deform $f$ by an isotopy so that $\left|T \cap T^{\prime}\right|$ is minimal. After cutting along $T$, we get a manifold $N=T \times I$, so if $\left|T \cap T^{\prime}\right|=0$, we can deform $f$ by an isotopy so that 
$f(T)=T$, and a further isotopy will deform $f$ to a torus fiber-preserving homeomorphism, and we are done. So we assume that $\left|T \cap T^{\prime}\right| \neq 0$.

By an innermost circle argument one can eliminate all trivial circles in $T \cap T^{\prime}$. Thus each component of $T^{\prime}-T$ is an annulus. Also, a boundary compression of an annulus of $T^{\prime}-T$ would deform $T^{\prime}$ to a torus having less intersection with $T$. Thus the minimality of $\left|T \cap T^{\prime}\right|$ implies that these annuli are $\partial$-incompressible in the manifold $N$ obtained by cutting $M$ along $T$, so they are essential annuli $P$ in $N$. There is an $S^{1}$-bundle structure of $N$ over an annulus $A$, so that $P$ is a union of fibers. $M$ can be recovered by gluing the two sides of $\partial N$ together. Since the boundary of $P$ matches each other to form the torus $T^{\prime}$, the $S^{1}$ bundle structure extends over $M$, whose orbifold $X(M)$ is obtained by gluing the two boundary components of $A$ together, hence is a torus.

If $M$ is a union of two twisted $I$-bundles over the Klein bottle glued together along their boundary, then there is a homeomorphism

$$
\rho: M \rightarrow J=[-1,1],
$$

such that $\rho^{-1}(x)$ is a Klein bottle if $x= \pm 1$, and is a torus otherwise. The map $\rho$ is called a torus bundle structure of $M$ over $J$, while $J$ is considered as a closed 1-orbifold with two singular points at \pm 1 . The proof of the following lemma is similar to that of Lemma 3.7. We omit the details.

Lemma 3.8. Let $\rho: M \rightarrow J$ be a torus bundle structure of $M$ over $J$. Let $f: M \rightarrow M$ be a homeomorphism. Then either $M$ is a Seifert fiber space over a Euclidean orbifold, or $f$ is isotopic to a torus fiber preserving homeomorphism with respect to the fibration $\rho$.

Lemma 3.9. Suppose $p: M \rightarrow X(M)$ is a Seifert fibration of a Euclidean manifold $M$ with orbifold $X(M)=S(2,4,4)$ or $S(2,2,2,2)$. Then any homeomorphism $f: M \rightarrow M$ is isotopic to a fiber-preserving homeomorphism with respect to this fibration.

Proof. First assume $X(M)=S(2,4,4)$. Put $T=S^{1} \times S^{1}$, with each point represented by $(x, y)$, where $x, y$ are mod 1 real numbers. Then the manifold $M$ can be expressed as $(T \times I) / \varphi$, with gluing map

$$
\varphi: T \times 0 \rightarrow T \times 1
$$


given by $\varphi(x, y, 0)=(-y, x, 1)$. Consider the universal covering $\widetilde{W}$ of $W=$ $T \times I$, which is identified with $\mathbb{R}^{2} \times I$. The lifting of the two order 4 singular fibers of $M$ in $\widetilde{W}$ are the vertical arcs at $(x, y)$, with both $x, y$ integers, or both half integers.

Claim 1. There is a fiber-preserving homeomorphism $f^{\prime}$ on $M$ which reverses the fiber orientation.

On $W$ the homeomorphism is given by $f^{\prime}(x, y, z)=(y, x, 1-z)$. Since

$$
f^{\prime} \circ \varphi(x, y, 0)=f^{\prime}(-y, x, 1)=(x,-y, 0)=\varphi^{-1} \circ f^{\prime}(x, y, 0),
$$

the map $f^{\prime}$ induces a homeomorphism on $M$, which clearly is fiber-preserving and fiber-orientation-reversing. The claim follows.

The product structure $W=T \times I$ gives a torus bundle structure of $M$ over $S^{1}$. By Lemma 3.7 we may assume that $f$ preserves the torus fibration. Note that the homeomorphism $f^{\prime}$ constructed in the claim is also torus fiberpreserving, and it reverses the orientation of $S^{1}$, the torus bundle orbifold. If $f$ also reverses the orientation of $S^{1}$, then $f^{\prime} \circ f$ is orientation-preserving on $S^{1}$, and $f$ is isotopic to a Seifert fiber-preserving homeomorphism if and only if $f^{\prime} \circ f$ is. Therefore, by considering $f^{\prime} \circ f$ instead if necessary, we may assume without loss of generality that $f$ preserves the orientation of $S^{1}$. After an isotopy, we may further assume that $f$ induces an identity map on $S^{1}$, so it maps each horizontal torus $T \times t$ to itself. We say that $f: M \rightarrow M$ is a level preserving map.

Let $\alpha: I \rightarrow M$ be given by $\alpha(t)=(0,0, t)$. It is a path representing a singular fiber. By an isotopy we may assume that $f$ fixes the base point of $\alpha$. Let $\alpha^{\prime}$ be the path $f \circ \alpha$.

Claim 2. The path $\alpha^{\prime}$ is isotopic to a singular fiber in $M$.

Let $\widetilde{\alpha}$ and $\widetilde{\alpha}^{\prime}$ be the lifting of $\alpha$ and $\alpha^{\prime}$ on $\widetilde{W}$ with initial point $(0,0,0)$. Let $\widetilde{\beta}^{\prime}$ be the straight line in $\widetilde{W}$ connecting the ends of $\widetilde{\alpha}^{\prime}$. Since $f$ is level preserving, $\widetilde{\alpha}^{\prime}$ intersect each plane $\mathbb{R}^{2} \times t$ at a single point, so there is an isotopy $\psi_{t}$ from $\widetilde{\alpha}^{\prime}$ to $\widetilde{\beta}^{\prime}$ rel $\partial$, moving each point of $\widetilde{\alpha}^{\prime}$ along the straight line on a level plane to the corresponding point of $\widetilde{\beta}^{\prime}$. For each $t$, the image of the path $\psi_{t}$ under the projection $q: \widetilde{W} \rightarrow M$ is a closed curve, which has no self intersection because $\psi_{t}$ intersects each level plane at a single point. Hence $\alpha^{\prime}$ is isotopic to the curve $\beta^{\prime}=q \circ \widetilde{\beta}^{\prime}$. Without loss of generality, we may assume that $\widetilde{\alpha}^{\prime}$ is a straight line, connecting $(0,0,0)$ to $(x, y, 1)$. Note that $x, y$ must be integers.

Put

$$
(\bar{x}, \bar{y})=\left(\frac{x-y}{2}, \frac{x+y}{2}\right) .
$$


For any $t$, let

$$
h_{t}: I \rightarrow \mathbb{R}^{2} \times I
$$

be the straight line from $t(\bar{x}, \bar{y}, 0)$ to the point $(1-t)(x, y, 1)+t(\bar{x}, \bar{y}, 1)$. Each $h_{t}$ projects to a simple arc in $T \times I$. By definition, we have

$$
\begin{aligned}
\varphi\left(h_{t}(0)\right) & =\varphi(t(\bar{x}, \bar{y}, 0))=t(-\bar{y}, \bar{x}, 1) \\
& =\left(\frac{t(-x-y)}{2}, \frac{t(x-y)}{2}, 1\right) \\
& =(1-t)(x, y, 1)+t(\bar{x}, \bar{y}, 1) \\
& =h_{t}(1) \quad \bmod 1,
\end{aligned}
$$

so each $h_{t}$ gives a closed curve in $M$. Moreover, since $h_{t}$ intersects each level torus at a single point, it has no self intersection. Thus $h_{t}$ is an isotopy of $\alpha^{\prime}$. When $t=1$, the loop $h_{1}$ is a vertical line $h_{1}(s)=(\bar{x}, \bar{y}, s)$, which is a singular fiber of $M$ because $(\bar{x}, \bar{y})$ are both integers or both half integers. This proves the claim. Figure 3.1 shows the isotopy in $\mathcal{R}^{2} \times I$, when $(x, y)=(1,0)$. The vertical line at $(0,0,0)$ is the curve $\widetilde{\alpha}$, the line from $(0,0,0)$ to $(1,0,1)$ is $\widetilde{\alpha}$, the vertical line at $(\bar{x}, \bar{y}, 0)=\left(\frac{1}{2}, \frac{1}{2}, 0\right)$ is the image of $h_{1}$, which is the lift of a singular fiber in $M$, and the lines between them represent the isotopy $h_{t}$.

The isotopy extends to an isotopy of $M$, modifying $f$ to a homeomorphism $g$, which maps the singular fiber $\alpha$ to another singular fiber. By Lemma 3.3 such a map is isotopic to a fiber-preserving homeomorphism.

The proof for the case $X(M)=S(2,2,2,2)$ is similar. The gluing map $\varphi: T \times 0 \rightarrow T \times 1$ is now given by $\varphi(x, y, 0)=(-x,-y, 1)$. Write $(\bar{x}, \bar{y})=$ $(x / 2, y / 2)$, and define $h_{t}$ using the same formula as above. The remaining part of the proof are the same, only notice that in this case a vertical line of $\widetilde{W}$ at $(x, y)$ represents a singular fiber of $M$ if and only if each of $x$ and $y$ is an integer or a half integer.

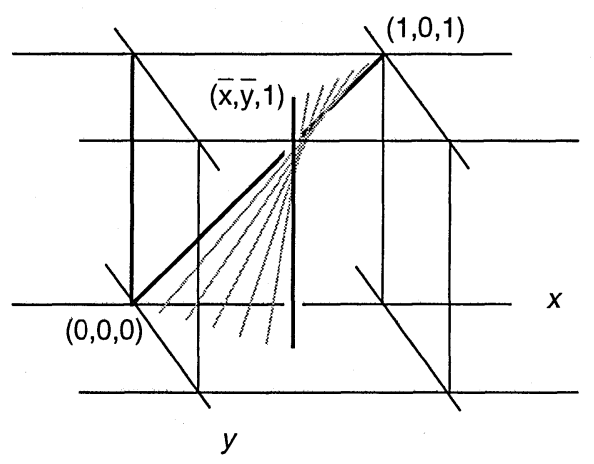

Figure 3.1. 
We now consider the manifold $M=S^{2} \times S^{1}$. Put $S^{2}=D_{1}^{2} \cup D_{2}^{2}$, and $V_{i}=D_{i}^{2} \times S^{1}$, so $M$ is the union of $V_{1}$ and $V_{2}$ along a torus $T$. Let $m, l$ be a meridian-longitude pair on $T$, where $m$ bounds a disk in both $V_{i}$. The torus $T$ is a Heegaard splitting torus of $M$, which is unique up to isotopy [Wa2]. Thus any homeomorphism $f: M \rightarrow M$ can be deformed by an isotopy so that $f(T)=T$. The homeomorphism $\left.f\right|_{T}$ corresponds to a matrix

$$
\left(\begin{array}{ll}
p & q \\
r & s
\end{array}\right)
$$

so that $f_{*}([l])=p[l]+q[m]$ and $f_{*}([m])=r[l]+s[m]$ in $H_{1}(T)$. Since $\left.f\right|_{T}$ extends over the solid tori, we have $r=0$ and $s= \pm 1$, so $p= \pm 1$. Note that any homeomorphism on $T$ extends to a unique orientation-preserving homeomorphism on $M$ up to isotopy, which maps each $V_{i}$ to itself if and only if the determinant of the matrix equals 1 .

Denote by $\alpha, \beta, \gamma$ the orientation-preserving homeomorphisms on $M$ corresponding to the following matrices, respectively.

$$
\left(\begin{array}{cc}
-1 & 0 \\
0 & -1
\end{array}\right),\left(\begin{array}{cc}
1 & 0 \\
0 & -1
\end{array}\right),\left(\begin{array}{cc}
1 & 1 \\
0 & -1
\end{array}\right)
$$

These matrices generate all the matrices satisfying the conditions that $r=0$ and $s= \pm 1$, hence the homeomorphisms $\alpha, \beta, \gamma$ generate the group $\mathcal{H}^{+}(M)$ of all isotopy classes of orientation-preserving homeomorphisms on $M$, which is an index 2 subgroup of the homeotopy group $\mathcal{H}(M)$.

There is an isotopy of $S^{2}$ which changes $m$ to $-m$, the product of which with the identity map on $S^{1}$ then deforms $\beta$ to the identity map, so $\beta$ represents the identity in $\mathcal{H}^{+}(M)$. Also, $\alpha$ and $\gamma$ both have order 2, and they commute, so they generate a group $\mathbb{Z}_{2}+\mathbb{Z}_{2}$.

Each non meridional curve on $T$ extends to a unique Seifert fibration of $M$. Consider the Seifert fibration corresponding to the curve $C$ which represents $[m]+2[l]$ in $H_{1}(T)$. Clearly, $\alpha$ maps the isotopy class of $C$ to itself, with orientation reversed. Since

$$
\gamma_{*}[C]=\gamma_{*}[m]+2 \gamma_{*}[l]=-[m]+2([l]+[m])=[C],
$$

we see that $\gamma$ also maps the isotopy class of $C$ to itself. Thus they both preserves the Seifert fibration corresponding to $C$. There are infinitely many isotopy classes of Seifert fibrations on $S^{2} \times S^{1}$, all of which are preserved by the map $\alpha$, but it is interesting that the fibration above is the only one preserved by $\gamma$. Let $\delta$ be the orientation-reversing involution of $M$ such that 
$\left.\delta\right|_{T}=i d$. Then $\delta$ also preserves all curves on $T$, hence all Seifert fibrations of $M$. Since $\alpha, \gamma, \delta$ generate the homeotopy group of $M$, it follows that the fibration corresponding to $C$ is preserved by all homeomorphisms on $M$. We have proved the following lemma for $S^{2} \times S^{1}$. Recall from [Sc, p. 457] that the two manifolds listed are the only orientable closed manifolds which admit the $S^{2} \times \mathbb{R}$ geometry.

Lemma 3.10. Suppose $M$ is either $S^{2} \times S^{1}$ or $R P^{3} \# R P^{3}$. Then there is a Seifert fibration on $M$ such that any homeomorphism on $M$ is isotopic to a fiber-preserving homeomorphism with respect to this fibration.

Proof. We have seen that the lemma is true for $S^{2} \times S^{1}$, so we assume that $M=R P^{3} \# R P^{3}$. Let

$$
\varphi=\varphi_{1} \times \varphi_{2}: S^{2} \times S^{1} \rightarrow S^{2} \times S^{1},
$$

where $\varphi_{1}$ is an antipodal map on $S^{2}$, and $\varphi_{2}: S^{1} \rightarrow S^{1}$ is given by the involution $\varphi_{2}(x, y)=(-x, y)$, with $S^{1}$ considered the unit circle in $R^{2}$. Then the manifold $M$ is a quotient

$$
M=\left(S^{2} \times S^{1}\right) / \varphi
$$

Let $\eta=\varphi_{1} \times i d$, and $\psi=\varphi_{1} \times \psi_{2}$, where $\psi_{2}(x, y)=(x,-y)$. Clearly, the maps $\eta$ and $\psi$ commute with $\varphi$, so they induce homeomorphisms $\widehat{\eta}, \widehat{\psi}: M \rightarrow$ $M$, which reverse the orientation of a reducing sphere in $M$. Moreover, the product $S^{1}$-bundle structure of $S^{2} \times S^{1}$ is preserved by all the three maps, so it induces a Seifert fibration on $M$, which is preserved by $\widehat{\eta}$ and $\widehat{\psi}$. The homeomorphism $\widehat{\eta}$ is orientation-reversing. Thus to prove the lemma, it remains to show that any orientation-preserving homeomorphism on $M$ is isotopic to either $\widehat{\psi}$ or the identity.

Let $S$ be an essential 2-sphere in $M$, which is unique up to isotopy because $M$ has only two irreducible prime factors, so any homeomorphism $f$ on $M$ can be deformed by an isotopy so that $f(S)=S$. Since $\widehat{\psi}$ reverses the orientation of $S$, exactly half of the isotopy classes of orientation-preserving homeomorphisms on $M$ preserve the orientation of $S$. Therefore, we need only show that if $f$ preserves the orientations of both $M$ and $S$, then $f$ is isotopic to the identity map. Since any orientation-preserving homeomorphism on $S$ is isotopic to the identity map, we may assume that $\left.f\right|_{S}=i d$. The sphere $S$ cuts $M$ into two pieces $X_{1}, X_{2}$, each being a punctured $R P^{3}$, which is a twisted $I$-bundle over the projective plane $P=R P^{2}$. Since $f$ 
is orientation-preserving, it maps each $X_{i}$ to itself. The result now follows from the following claim.

Claim. If $X$ is a twisted I-bundle over the projective plane $P$, and $f$ : $X \rightarrow X$ is an orientation-preserving homeomorphism such that $\left.f\right|_{\partial X}=i d$, then $f$ is isotopic rel $\partial X$ to the identity map.

Clearly, the projective plane $P$ in $X$ is unique up to isotopy. We can first deform $f$ so that $f$ sends $P$ to itself, then further isotop $f$ so that the restriction of $f$ on $P$ is the identity. where

Put $Y=S^{2} \times[0,1]$. View $X$ as the quotient space of $\left(S^{2} \times[0,1]\right) / \eta$,

$$
\eta: S^{2} \times 1 \rightarrow S^{2} \times 1
$$

identifies each point on the sphere $S^{2} \times 1$ to its antipodal point. Let $\tilde{f}: Y \rightarrow$ $Y$ be the lift of $f$. Now the restriction of $\tilde{f}$ on $S^{2} \times 0$ is the identity, and the restriction of $\tilde{f}$ on $S^{2} \times 1$ is the lifting of the identity map on $P$, hence is either the identity or the antipodal map; but since it is the restriction of the orientation-preserving homeomorphism $\widetilde{f}$ on a boundary component of $Y$, it can not be the antipodal map. Thus the restriction of $\widetilde{f}$ on $\partial Y$ is the identity.

Consider $S^{2}$ as the standard unit sphere in $\mathbb{R}^{3}$, and let $x$ be the north pole. Then $C=x \times[0,1]$ is an arc in $Y$. By the Light Bulb Theorem [Ro, page 257], we may change $\widetilde{f}$ by isotopy rel $\partial Y$ so that $\left.\widetilde{f}\right|_{C}=i d$. Let $D_{1}, D_{2}$ be the upper and lower half disks of $S^{2}$. Deform $\tilde{f}$ by an isotopy rel $\partial Y$ so that it maps each $D_{1} \times t$ isometrically to itself, then further deform $\tilde{f}$ on $D_{2} \times[0,1]$, rel $\partial$, so that it maps each $D_{2} \times t$ isometrically to itself. We can now assume that $\tilde{f}$ maps each $S^{2} \times t$ to itself isometrically. In particular, it always maps a pair of antipodal points of $S^{2} \times t$ to another pair of antipodal points.

Let $\widetilde{f}_{t}: S^{2} \rightarrow S^{2}$ be the restriction of $\tilde{f}$ on the level sphere $S^{2} \times t$, i.e, $\widetilde{f}_{t}$ satisfies

$$
\left.\widetilde{f}(u, t)=\left(\widetilde{f_{t}}(u), t\right)\right) .
$$

Define an isotopy $g_{s}$ on $Y=S^{2} \times[0,1]$ by

$$
g_{s}(u, t)=\left\{\begin{array}{cc}
(u, t) & \text { if } t \leq s \\
\left(\widetilde{f}_{t-s}(u), t\right) & \text { if } t \geq s
\end{array} .\right.
$$

Then $g_{s}$ is an isotopy from $\tilde{f}$ to the identity map, rel $S^{2} \times 0$. Moreover, the restriction of $g_{s}$ to $S^{2} \times 1$ is always an isometry, hence maps antipodal 
pairs to antipodal pairs. Therefore, $g_{s}$ induces an isotopy $h_{s}$ on the quotient space $X=Y / \eta$, deforming $f$ to the identity map rel $\partial X$.

Recall from [Sc, p. 446] that there is a unique orientable Euclidean manifold which admits a Seifert fibration with orbifold $P(2,2)$. We denote this manifold by $M_{P(2,2)}$. The following theorem is sharp: Each of the four manifolds listed admits some homeomorphism which does not preserve any Seifert fibration of $M$.

Theorem 3.11. Suppose $M$ is a compact orientable Seifert fiber space which is not $T^{3}, M_{P(2,2)}, S^{1} \times D^{2}$, or $T \times I$. Then there is a Seifert fibration $p: M \rightarrow X(M)$, so that any orientation-preserving homeomorphism on $M$ is isotopic to a fiber-preserving homeomorphism with respect to this fibration. Moreover, if $M$ is not a lens space or $S^{3}$, then the result is true for all homeomorphisms on $M$.

Proof. By the remark after Theorem 3.1, we need only consider manifolds covered by $T^{3}, S^{3}$ or $S^{2} \times \mathbb{R}$. The only manifolds covered by $S^{2} \times \mathbb{R}$ are $S^{2} \times S^{1}$ and $R P^{3} \# R P^{3}$, in which case the result follows from Lemma 3.10. So we assume that $M$ is covered by $T^{3}$ or $S^{3}$, i.e., it is a Euclidean or spherical manifold.

If $M=S^{3}$, all orientation-preserving homeomorphisms are isotopic to the identity, which preserves any Seifert fibration of $S^{3}$. Lemma 3.4 covers lens spaces. The remaining manifolds have Seifert fibrations $p: M \rightarrow X(M)$ with orbifolds $X(M)=S(p, q, r)$, or $S(2,2,2,2)$, or $P(2,2)$, or $T^{2}$. The case $X(M)=S(2,2,2,2)$ is proved in Lemma 3.9. The last two cases are excluded from the assumption of the theorem. In the remaining cases, $X(M)=S(p, q, r)$. Since $M$ is Euclidean or spherical, we have

$$
\frac{1}{p}+\frac{1}{q}+\frac{1}{r} \geq 1
$$

If $(p, q, r)=(2,2, k)$, or $(2,3,4)$, or $(2,4,4)$, this is covered by lemmas 3.5 , 3.6 and 3.9 , respectively. In the remaining cases, either $(p, q, r)=(3,3, k)$ for some $k \geq 2$, or $(p, q, r)=(2,3, k)$ for $k \geq 5$, which have been covered by Theorem 3.2.

According to [Ja, Theorem VI.16], the following orientable manifolds admit different Seifert fibrations up to isomorphism.

(a) Lens spaces (including $S^{3}$ and $S^{2} \times S^{1}$ ). 
(b) Prism manifolds, i.e. Seifert fiber spaces with orbifolds $X(M)=$ $S(2,2, p)$.

(c) Double of twisted $I$-bundle over the Klein bottle, i.e. a Euclidean Seifert fiber space $M$ with $X(M)=S(2,2,2,2)$.

(d) The solid torus.

(e) The twisted $I$-bundle over the Klein bottle.

The following result supplements Theorems 3.1 and 3.2.

Corollary 3.12. Let $M$ be a compact orientable Seifert fiber space. Then the Seifert fibration of $M$ is unique up to isotopy if and only if $M$ is not $T^{3}$, $M_{P(2,2)}, T \times I$, or one of the manifolds listed above.

Proof. Suppose $M$ is not one of the manifolds listed above. Then by [Ja, Theorem VI.17], Seifert fibrations of $M$ are unique up to isomorphism, that is, if $p_{i}: M \rightarrow X_{i}$ are Seifert fibrations for $i=1,2$, then $X_{1}=X_{2}$, and there is a homeomorphism $f: M \rightarrow M$, such that $p_{2} \circ f=p_{1}$. If $M$ is not $T^{3}, T \times I$ or $M_{P(2,2)}$ either, then by Theorem 3.11, up to isotopy we may assume that the homeomorphism $f$ is a fiber-preserving map with respect to the fibration $p_{1}$. It follows that the two fibrations are the same up to isotopy.

Conversely, if $M$ is one of the manifolds listed above, then it has non isomorphic Seifert fibrations, so of course they are not isotopic. If $M$ is $T^{3}$ or $T \times I$, then any simple closed curve on $T \times 0$ is a regular fiber of some Seifert fibration of $M$. Since these curves represent different elements in $H_{1}(M)$, the fibrations are different, so $M$ has infinitely many non isotopic fibrations. Finally for the manifold $M_{P(2,2)}$, it will be shown in Proposition 4.2 that it has exactly three non isotopic Seifert fibrations.

\section{Homeomorphisms on $M_{P(2,2)}$.}

There are two closed manifolds which are excluded in the statement of Theorem 3.11. One is $T^{3}$, the other is the manifold $M_{P(2,2)}$, which is the Euclidean manifold with a Seifert fibration over the orbifold $P(2,2)$. Homeomorphisms on $T^{3}$ are clear: Any homeomorphism of $T^{3}$ is isotopic to an affine map; see the proof of Lemma 8.3. The following theorem covers the case of $M_{P(2,2)}$.

Theorem 4.1. Any homeomorphism $f$ on the manifold $M=M_{P(2,2)}$ is isotopic to an isometry with respect to a Euclidean metric. 
Proof. We would like to thank the referee for pointing out that this is a well known result. It can be found in [CV, p. 478] and [BZ, p. 195]. See the proof of the following proposition for more details.

Proposition 4.2. The manifold $M=M_{P(2,2)}$ has exactly three Seifert fibrations up to isotopy.

Proof. The euclidean structure of $M$ is described in [BZ] as follows. Consider the three euclidean isometries $\alpha_{1}, \alpha_{2}, \alpha_{3}$ of $\mathbb{R}^{3}$, which are $\pi$ rotations along the lines $l_{1}=\{x=1 / 2, y=1\}, l_{2}=\{y=1 / 2, z=1\}$ and $l_{3}=\{z=$ $1 / 2, x=1\}$. These rotations generate a group $O(L)$ whose quotient is $S^{3}$, in which the images of the $l_{i}$ form the Borromean ring $L$. Thus the quotient map $\mathbb{R}^{3} \rightarrow S^{3}$ induces a euclidean orbifold structure on $S^{3}$ with cone angle $\pi$ at $L$. Now $M$ is the double branched cover of $S^{3}$ branched along $L$, hence it is the quotient of $\mathbb{R}^{3}$ by the subgroup $\pi$ of $O(L)$ consisting of all elements which are the products of an even number of the $\alpha_{i}{ }^{\prime}$ s. This gives the euclidean structure of $M$.

The set of lines parallel to a given coordinate axis is invariant under the action of $\pi$, and their images in $M$ are circles, hence form a Seifert fibration of $M$. Thus there are three Seifert fibrations $\xi_{1}, \xi_{2}, \xi_{3}$ corresponding to the three coordinate axes of $\mathbb{R}^{3}$. These are not isotopic: If two Seifert fibrations are isotopic, then a regular fiber of one fibration is isotopic to that of another, so their liftings in the universal covering $\mathbb{R}^{3}$ can be deformed to each other by an isotopy such that the lengths of the traces of points are bounded above; in particular, they must have the same limit set on $S_{\infty}^{2}$, the sphere at the infinity. Since the coordinate axes have different limit points on $S_{\infty}^{2}$, the $\xi_{i}$ 's are mutually distinct. It remains to show that these are the only Seifert fibrations.

The homeotopy group $\mathcal{H}(M)$ of $M$ has order 96 (see [CV, p. 478]). Its elements are represented by isometries of $M$ with respect to the above euclidean metric. Boileau and Zimmermann [BZ, p. 195] gave an explicit description of the elements of this group. Its generators lift to the following isometries of $\mathbb{R}^{3}$ : (i) the involution $\alpha_{1}$; (ii) a $\pi$-rotation along the axis $\{x=y=1 / 2\}$; (iii) a $2 \pi / 3$-rotation along the axis $\{x=y=z\}$; (iv) a reflection along the plane $z=1 / 2 ;(\mathrm{v})$ a translation moving the origin to $(1 / 2,1 / 2,1 / 2)$, followed by a $\pi / 2$-rotation along the line $\{x=y=1 / 2\}$. Note that (ii)-(iv) generate a subgroup of order 24 of the symmetries of the unit cube, which together with the map in (v) induce the order 48 symmetry group of $\left(S^{3}, L\right)$, which is an index 2 subgroup of $\mathcal{H}(M)$. 
Clearly each of the above maps sends the three Seifert fibrations $\xi_{i}$ to themselves, with possibly a permutation. Let $\xi$ be a Seifert fibration of $M$. By [Ja, Theorem VI.17], Seifert fibrations of $M$ are unique up to isomorphism, so there is a homeomorphism $f: M \rightarrow M$ sending $\xi_{i}$ to some $\xi$. Since $f$ is isotopic to an isometry as above, which sends $\xi_{i}$ to some $\xi_{j}$, it follows that $\xi$ is isotopic to $\xi_{j}$.

\section{Fixed points and standard maps.}

Suppose $B^{n}$ is the unit ball in $\mathbb{R}^{n}$, and $f: B^{n} \rightarrow \mathbb{R}^{n}$ is a map with the origin $x$ as the only fixed point. Then the fixed point index of $x$ is defined as

$$
\text { ind }(f, x)=\text { degree }(\varphi) \text {, }
$$

where the map

$$
\varphi: S^{n-1}=\partial B^{n} \rightarrow S^{n-1}
$$

is defined by

$$
\varphi(y)=\frac{y-f(y)}{\|y-f(y)\|} .
$$

Since a manifold is locally Euclidean, this defines ind $(f, x)$ for isolated fixed points of maps $f: M \rightarrow M$ on closed manifolds.

Suppose $f$ is a map on a closed manifold $M$, and $C$ is a union of some components of Fix $(f)$. Then $C$ is compact, and there is an open neighborhood $V$ of $C$ containing no other fixed point of $f$. Perturb $f$ inside $V$ to a map $g$ so that it has only isolated fixed points in $V$. Then ind $(f, C)$ can be defined as the sum of ind $(g, x)$ over all fixed points of $g$ in $V$. It can be shown that this is independent of the perturbation.

Two fixed points $x, y$ of a map $f: M \rightarrow M$ are equivalent if there is a path $\gamma$ from $x$ to $y$, such that $f \circ \gamma$ and $\gamma$ are rel $\partial$ homotopic. In particular, all points in a component of Fix $(f)$ are equivalent. An equivalent class $C$ of fixed points of $f$ is called a Nielsen class. A Nielsen class $C$ is essential if ind $(f, C) \neq 0$; otherwise it is inessential. The Nielsen number $N(f)$ is defined as the number of essential fixed point classes in Fix $(f)$. The crucial property of $N(f)$ is that it is a homotopy invariant. In particular, it gives a lower bound for the number of fixed points for all maps homotopic to $f$. 


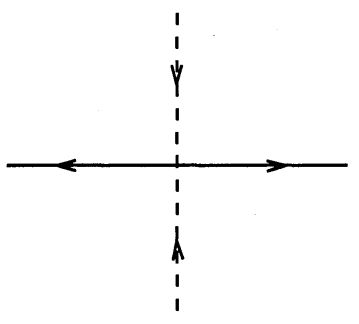

(a)

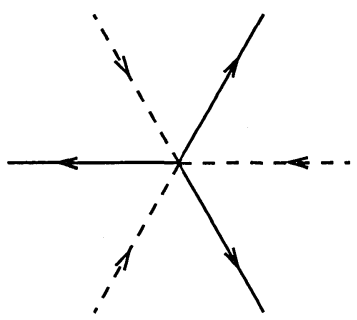

(b)

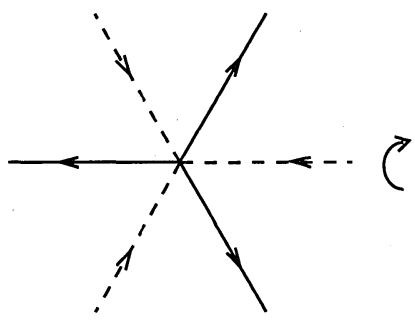

(c)

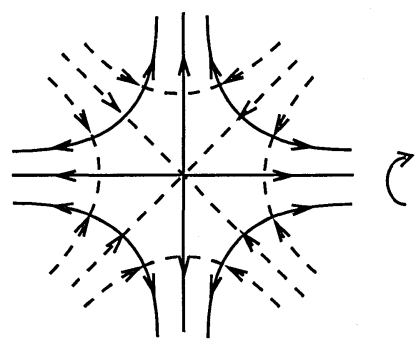

(d)

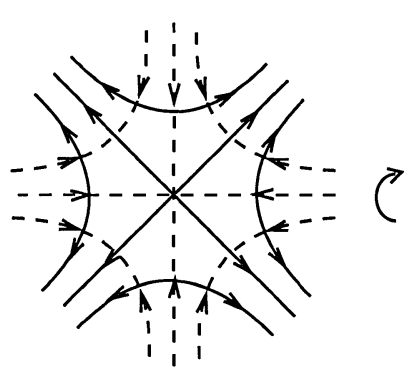

(e)

Figure 5.1.

Example 5.1. A local homeomorphism near an isolated fixed point $x$ of $f$ is of pseudo-Anosov type if it is the same as the restriction of a pseudoAnosov map near a fixed point. Figure 5.1 shows the picture of some pseudoAnosov type local homeomorphisms. In Figure 5.1(a), the homeomorphism is a linear map, expanding along the solid line direction, and shrinking along the dotted line direction. One can compute that ind $(f, x)=-1$. More generally, if $f$ is a pseudo-Anosov type homeomorphism near $x$, as shown in Figure 5.1(b), then ind $(f, x)=1-p$, where $p$ is the number of prongs of the singular foliation at $x$. If $f$ is the composition of the above map with a rotation sending a prong to another, the index is 1 . In Figure 5.1(c), (d) and (e), the homeomorphisms are orientation-reversing. These are the compositions of maps shown in Figure 5.1(b) and a reflection along an invariant line $l$, which is the union of two singular leaves of the invariant foliations of $f$. The index is 0 if the prong number $p$ is odd (Figure 5.1(c)), -1 if $p$ is even and the invariant line is a solid line (Figure 5.1(d)), and 1 otherwise (Figure 5.1(e)). See [JG, Lemma 3.6] for more details. Similarly, a 
local homeomorphism in a neighborhood of a boundary point is of pseudoAnosov type if it is the restriction of a global pseudo-Anosov map in a neighborhood of a boundary fixed point.

The combination of the above example and the following product formula will be useful in computing the index of fixed points for homeomorphisms of 3-manifolds. See [J1, Lemma I.3.5(iv)] for a proof.

Lemma 5.2. Suppose that $U_{i}$ is an open neighborhood of $x_{i}$ in a compact topological space $X_{i}$, and $f_{i}: U_{i} \rightarrow X_{i}$ is a homeomorphism with $x_{i}$ as the only fixed point. Let $x=\left(x_{1}, x_{2}\right)$, and let $f$ be the product map $f=f_{1} \times f_{2}$ : $X_{1} \times X_{2} \rightarrow X_{1} \times X_{2}$. Then

$$
\text { ind }(f, x)=\operatorname{ind}\left(f_{1}, x_{1}\right) \times \text { ind }\left(f_{2}, x_{2}\right) \text {. }
$$

If $A, B$ are subsets of $M$, then $\gamma: I \rightarrow M$ is a path from $A$ to $B$ if $\gamma(0) \in A$, and $\gamma(1) \in B$. If $\gamma_{0}, \gamma_{1}$ are paths from $A$ to $B$, and there is a homotopy $\varphi_{t}: I \rightarrow M$ from $\gamma_{0}$ to $\gamma_{1}$ such that $\varphi_{t}(0) \in A$ and $\varphi_{t}(1) \in B$ for all $t$, then we say that $\gamma_{0}$ is $(A, B)$ homotopic to $\gamma_{1}$, and write

$$
\gamma_{0} \sim \gamma_{1} \quad \operatorname{rel}(A, B) .
$$

When $A, B$ are one point sets, this is also written as

$$
\gamma_{0} \sim \gamma_{1} \quad \text { rel } \partial .
$$

A set $B$ is $f$-invariant if $f(B)=B$. Suppose $f: M \rightarrow M$ is a map, and $A, B$ are $f$-invariant sets of $M$. If there is a path $\gamma$ from $A$ to $B$ such that $\gamma \sim f \circ \gamma \operatorname{rel}(A, B)$, then we say that $A, B$ are $f$-related, denoted by

$$
A \sim_{f} B .
$$

In particular, two fixed points $x$ and $y$ of a map $f$ are in the same fixed point class if and only if they are $f$-related.

A covering $p: \widetilde{M} \rightarrow M$ is characteristic if the corresponding subgroup $p_{*} \pi_{1} \widetilde{M}$ is a characteristic subgroup of $\pi_{1} M$, i.e, it is invariant under any automorphism of $\pi_{1} M$. Recall that if $G$ is a finite index subgroup of a finitely generated group $H$, then there is a finite index characteristic subgroup $G^{\prime}$ of $H$, such that $G^{\prime} \subset G$. It follows that given any finite covering $p: \widetilde{M} \rightarrow$ $M$ of compact 3-manifold, there is a finite covering $q: \widehat{M} \rightarrow \widetilde{M}$ so that $p \circ q: \widehat{M} \rightarrow M$ is a characteristic covering. Notice that if $p: \widetilde{M} \rightarrow M$ is a characteristic covering, then for any homeomorphism $f: M \rightarrow M$ with $y=f(x)$, and any points $\widetilde{x}$ and $\widetilde{y}$ covering $x$ and $y$ respectively, there is a lifting $\widetilde{f}$ of $f$ such that $\widetilde{f}(\widetilde{x})=\widetilde{y}$. 
Lemma 5.3. Suppose that $A$ and $B$ are $f$-invariant subsets of $M$ which are $f$-related by a path $\gamma$. Let $p: \widetilde{M} \rightarrow M$ be a characteristic covering map, and $\widetilde{A}$ a component of $p^{-1}(A)$. Then there is a lifting $\widetilde{f}$ of $f$, and a lifting $\widetilde{\gamma}$ of $\gamma$, which $\widetilde{f}$-relates $\widetilde{A}$ to a component $\widetilde{B}$ of $p^{-1}(B)$.

Proof. First choose $\widetilde{\gamma}$ that covers $\gamma$. The homotopy $h_{t}: \gamma \sim f \circ \gamma$ lifts to a homotopy $\widetilde{h}_{t}$ on $\widetilde{M}$, with $\widetilde{h}_{0}(0)=\widetilde{\gamma}(0)$. Then $p \circ \widetilde{h}_{1}(0)=h_{1}(0)=f \circ \gamma(0)$, so $\widetilde{h}_{1}(0) \in p^{-1}(f \circ \gamma(0))$. Since $p$ is characteristic, there is a lifting $\widetilde{f}$ of $f$ such that $\widetilde{f}(\widetilde{\gamma}(0))=\widetilde{h}_{1}(0)$. Thus $\widetilde{h}_{t}$ is a homotopy $\widetilde{\gamma} \sim \widetilde{f} \circ \widetilde{\gamma}$ rel $(\widetilde{A}, \widetilde{B})$, where $\widetilde{B}$ is the component of $p^{-1}(B)$ containing $\widetilde{\gamma}(1)$.

Definition 5.4. A normal structure on a properly embedded arc or circle $C$ in a 3-manifold $M$ is an identification of a neighborhood $N(C)$ with $C \times D$, where $D$ is a disk, such that $C$ is identified with $C \times 0$. Suppose $C$ is a proper 1-dimensional component of $F i x(f)$. We say that $f$ preserves a normal structure on $C$ if there is a normal structure $N(C)=C \times D$ on $C$, such that $f$ restricted to a smaller neighborhood $N_{1}(C)=C \times D_{1}$ has the property that $f\left(x \times D_{1}\right) \subset x \times D$ for any $x \in C$.

Lemma 5.5. Suppose $f: M \rightarrow M$ is an orientation-preserving homeomorphism. If $C$ is a circle component of $\operatorname{Fix}(f)$, and $f$ preserves a normal structure on $C$, then $f$ can be deformed by an isotopy in a neighborhood of $C$ to a homeomorphism $g$ such that $\operatorname{Fix}(g)=\operatorname{Fix}(f)-C$.

Proof. Let $N_{1}(C)=C \times D_{1}$ be as in Definition 5.4. Identify $f\left(N_{1}(C)\right)$ with $S^{1} \times D^{\prime}$, where $D^{\prime}$ is the unit disc. Define an isotopy of $M$ by $\varphi_{t}(x, p)=$ $(x+t(1-\|p\|), p)$ on $f\left(N_{1}(C)\right)$ and identity outside, where $\|p\|$ is the norm of $p$. Since $f$ maps each $x \times D^{\prime}$ into $x \times D$, the composition $g=\varphi_{1} \circ f$ has no fixed points in $N_{1}(C)$.

Definition 5.6. A homeomorphism $f: M \rightarrow M$ is said to be of flipped pseudo-Anosov type at an isolated fixed point $x$ if there is a neighborhood $N(x)=D \times I$ of $x$, such that the restriction of $f$ on a smaller neighborhood $D_{1} \times I$ is a product $f_{1} \times f_{2}$, where $f_{1}$ is a pseudo-Anosov type map given in Example 5.1, and $f_{2}$ is the involution $f_{2}(t)=1-t$.

Notice that in this case, if we write $x=\left(x_{1}, x_{2}\right)$, then by Lemma 5.2 we have ind $(f, x)=\operatorname{ind}\left(f_{1}, x_{1}\right) \cdot$ ind $\left(f_{2}, x_{2}\right)=\operatorname{ind}\left(f_{1}, x_{1}\right)$, because in this case ind $\left(f_{2}, x_{2}\right)=1$. 
Lemma 5.7. Suppose $f: M \rightarrow M$ is an orientation-preserving homeomorphism. If $f$ is of flipped pseudo-Anosov type at some fixed point $x$ of $f$ in the interior of $M$, and ind $(f, x)=0$, then $f$ can be deformed by an isotopy in a neighborhood of $x$ to a homeomorphism $g$ such that $\operatorname{Fix}(g)=\operatorname{Fix}(f)-x$.

Proof. Let $f=f_{1} \times f_{2}: D_{1} \times I \rightarrow D \times I$ be as in the definition, and let $x=\left(x_{1}, x_{2}\right)$. Then ind $\left(f_{1}, x_{1}\right)=$ ind $(f, x)=0$. By [JG, $\S 2.2$, Remark 2], $f_{1}$ is rel $\partial D_{1}$ isotopic to a homeomorphism $g_{1}$ which has no fixed point. Extend this isotopy to an isotopy of $f$ in $D_{1} \times I$, rel $\partial\left(D_{1} \times I\right)$. Since $f$ exchanges the two sides of $D_{1} \times 0$, this will not create new fixed points.

Definition 5.8. Suppose $M$ is a compact 3-manifold with boundary consisting of tori. A homeomorphism $f: M \rightarrow M$ is standard on the boundary if for any component $T$ of $\partial M$, the map $\left.f\right|_{T}$ is of one of the following types.

(i) A fixed point free map.

(ii) A periodic map with isolated fixed points.

(iii) A fiber-preserving, fiber-orientation-reversing map with respect to some $S^{1}$ fibration of $T$, inducing an involution on the orbifold; moreover, $\left.f\right|_{T}$ is not isotopic to a map of the above two types.

Definition 5.9. Suppose $M$ is a compact 3-manifold with boundary consisting of tori.

(1) A homeomorphism $f$ on a compact 3-manifold $M$ is said to have the FR-property (Fixed-point Relating Property) if the following is true:

If $A \in \operatorname{Fix}(f)$ and $B$ is either a fixed point of $f$ or an $f$-invariant component of $\partial F$, and $A, B$ are $f$-related by a path $\gamma$, then $\gamma$ is $(A, B)$ homotopic to a path in Fix $(f)$.

(2) $f$ is a Type 1 standard map if (i) $f$ has the FR-property, (ii) $f$ is standard on the boundary, (iii) Fix $(f)$ consists of isolated points, and (iv) $f$ is of flipped pseudo-Anosov type at each fixed point.

(3) $f$ is a Type 2 standard map if it satisfies (i), (ii) above, as well as (iii) Fix $(f)$ is a properly embedded 1-dimensional submanifold, and (iv) $f$ preserves a normal structure on Fix $(f)$. 
Lemma 5.10. Suppose $M$ is a closed 3-manifold. If $f: M \rightarrow M$ is a type 1 standard map, then $f$ is isotopic to a homeomorphism $g$ with exactly $N(f)$ fixed points. If $f$ is a type 2 standard map, then it is isotopic to a fixed point free homeomorphism.

Proof. The FR-property implies that each fixed point class of $f$ is path connected. In particular, if $f$ is a Type 1 standard map on a closed manifold, then each fixed point is a Nielsen class, and by Lemma $5.7 f$ can be modified by an isotopy supported in a neighborhood of inessential fixed points to eliminate these fixed points, deforming $f$ to a homeomorphism $g$ with exactly $N(f)$ fixed points. If $f$ is a type 2 standard map, since $M$ is closed, all components of $\operatorname{Fix}(f)$ are circles, which can be eliminated using Lemma 5.5 .

The following result about surface homeomorphisms is due to Jiang and Guo [JG, Lemma 1.2 and Lemma 2.2] when $F$ is hyperbolic. The result is well known if $F$ is Euclidean or spherical, and can be proved in the same way.

Lemma 5.11. (1) If $\varphi: F \rightarrow F$ is a periodic map on a surface, then it has the FR-property, except when $F=S^{2}$ and $\varphi$ is orientation preserving.

(2) If $F$ is hyperbolic or Euclidean, and $\varphi: F \rightarrow F$ is a pseudo-Anosov or Anosov map, then each fixed point of $\varphi$ is an isolated point, and it has the FR-property, except that two fixed points on the same boundary component $C$ of $F$ are $\varphi$-related if $\varphi$ is isotopic to id on $C$.

(3) In particular, if $F$ is orientable and $\varphi$ is orientation reversing, then in both cases (1) and (2), $\varphi$ has the FR-property.

Lemma 5.12. Suppose $f$ is an orientation-reversing homeomorphism on a torus $T$. Then $f$ is isotopic to either a fixed point free homeomorphism or an Anosov map.

Proof. By an isotopy we may assume that $f$ is either periodic, reducible, or Anosov. If it is periodic and Fix $(f)$ is nonempty, then it is an involution, so it is also reducible. For a reducible homeomorphism, up to isotopy we may assume that its fixed point set consists of two circles, which can be eliminated by a small flow along the circle direction. 
Remark 5.13. Whether two fixed points of a map are equivalent is a global property. It may happen that $f$ maps a submanifold $M_{1}$ of $M$ to itself, and $f$ has two fixed points $x, y$ in $M_{1}$ such that they are inequivalent in $M_{1}$, but equivalent in $M$. Here is an example. Let $F_{1}, F_{2}$ be once punctured tori, let $f_{1}: F_{1} \rightarrow F_{1}$ be an orientation reversing pseudo-Anosov map, and let $f_{2}: F_{2} \rightarrow F_{2}$ be an orientation reversing involution. Glue $f_{1}$ and $f_{2}$ together along the boundary $\partial F_{1}=\partial F_{2}$ to get a map $f$ on $F=F_{1} \cup F_{2}$. Then the two fixed points of $f_{1}$ on $\partial F_{1}$ are inequivalent in $F_{1}$, but equivalent in $F$. Now let $M=F \times S^{1}$, and let $g: M \rightarrow M$ be the product $f \times r$, where $r$ is an involution on $S^{1}$. Then $g$ is an orientation-preserving map. Let $g_{1}$ be the restriction of $g$ on $M_{1}=F_{1} \times S^{1}$. Then the four fixed points of $g_{1}$ on $\partial M_{1}$ are inequivalent in $M_{1}$, but as fixed points of $g$, they form two equivalent pairs in $M$.

\section{Fiber-preserving homeomorphisms.}

Let $f: M \rightarrow M$ be an orientation-preserving, fiber-preserving homeomorphism on a Seifert fiber space $M$, let $p: M \rightarrow X(M)$ be the projection map, and let $\widehat{f}: X(M) \rightarrow X(M)$ be the induced homeomorphism on the orbifold. In this section we will consider the case that $\widehat{f}: X(M) \rightarrow X(M)$ is either periodic, Anosov, or pseudo-Anosov.

Consider the restriction of $f$ on $p^{-1}(W)$ for a component $W$ of Fix $(\widehat{f})$. For each $x \in W$, the fiber $L_{x}=p^{-1}(x)$ is $f$-invariant. Since $W$ is connected, it is clear that $f$ preserves the orientation of a fiber over $W$ if and only if it preserves the orientation of all fibers over $W$. In this case we say that $f$ is fiber-orientation-preserving over $W$. Otherwise it is fiber orientationreversing over $W$.

Lemma 6.1. Suppose $f$ is fiber-orientation-preserving over some component $W$ of $\operatorname{Fix}(\widehat{f})$. Let $M^{\prime}=p^{-1}(N(W))$. Then $f$ can be deformed via a fiber-preserving isotopy rel $M-M^{\prime}$, to a homeomorphism which has no fixed point in $M^{\prime}$.

Proof. By a small fiber-preserving isotopy rel $M-M^{\prime}$ we may modify $f$ to a homeomorphism $g$ such that the orbifold homeomorphism $\widehat{g}$ has only isolated fixed points in $N(W)$. Thus $g$ maps only finitely many fibers $C_{1}, \ldots C_{n}$ of $M^{\prime}$ to themselves.

For each $C_{i}$, since $g$ is fiber-orientation-preserving, $\left.g\right|_{C_{i}}$ can be deformed by an isotopy to a fixed point free homeomorphism. The isotopy can be 
extended to a fiberwise isotopy supported in a small neighborhood of $C_{i}$, eliminating all fixed points in $N\left(C_{i}\right)$. The result follows by performing such an isotopy for each $C_{i}$.

Notice that if $M$ is a connected orientable circle bundle over an orientable surface, then all fibers can be coherently oriented, so a fiber-preserving homeomorphism $f: M \rightarrow M$ either preserves or reverses the orientation on all fibers.

Lemma 6.2. Suppose $q: M \rightarrow F$ is a connected orientable circle bundle over an orientable compact surface $F$, and suppose $f: M \rightarrow M$ is an orientation-preserving, fiber-orientation-reversing homeomorphism, such that $\widehat{f}: X(M) \rightarrow X(M)$ is periodic, Anosov or pseudo-Anosov. Then $f$ has the FR-property.

Proof. Consider the case that $A, B$ are fixed points of $f$, and are $f$-related by a path $\gamma$. (The case that $B$ is an $f$-invariant boundary component of $f$ is similar.) Then $\widehat{A}=q(A)$ and $\widehat{B}=q(B)$ are fixed points of $\widehat{f}$ on $X(M)$, and they are $\widehat{f}$-related by $\widehat{\gamma}=q \circ \gamma$. Since $f$ is fiber orientation-reversing, $\widehat{f}$ is orientation-reversing on $F$. Hence by Lemma $5.11, \widehat{\gamma}$ is $(\widehat{A}, \widehat{B})$ homotopic to a path $\widehat{\beta}$ in $\operatorname{Fix}(\widehat{f})$. As $f$ is fiber orientation-reversing, $\operatorname{Fix}(\widehat{f})$ is double covered by $\operatorname{Fix}(f)$, so there is a unique path $\beta$ in $\operatorname{Fix}(f)$ covering $\widehat{\beta}$, with $\beta(0)=\gamma(0)$. The path $\beta^{-1} \cdot \gamma$ projects to the null-homotopic loop $\widehat{\beta}^{-1} \cdot \widehat{\gamma}$ on $F$, so it is rel $\partial$ homotopic to a path $\alpha$ in the fiber $L$ over $\widehat{\gamma}(1)$. Since

$$
\alpha \sim \beta^{-1} \cdot \gamma \sim f \circ\left(\beta^{-1} \cdot \gamma\right) \sim f \circ \alpha \quad \text { rel } \partial,
$$

and since $f$ reverses the orientation of $L$, this is true only if $\alpha$ is a nullhomotopic loop in $L$. It follows that $\gamma \sim \beta$ rel $\partial$.

Proposition 6.3. Suppose $f: M \rightarrow M$ is an orientation preserving homeomorphism which is fiber preserving with respect to a Seifert fibration of $M$.

(1) If $X(M)$ is hyperbolic or Euclidean, and $\widehat{f}: X(M) \rightarrow X(M)$ is pseudoAnosov or Anosov, then $f$ is isotopic to a Type 1 standard map.

(2) If $\widehat{f}: X(M) \rightarrow X(M)$ is periodic, then $f$ is isotopic to a Type 2 standard map. 
Proof. By Lemma 6.1 we may assume that $f$ reverses the orientation of any fiber which contains a fixed point of $f$. In particular, if $T$ is a component of $\partial M$, then either $\left.f\right|_{T}$ is fixed point free, or it is a fiber-preserving, fiberorientation-reversing map. Hence up to a fiber-preserving isotopy we may assume that $f$ is standard on the boundary.

Suppose $A \in F i x(f)$ is $f$-related to $B$ by a path $\gamma$, where $B$ is a fixed point of $f$ or an $f$-invariant component of $\partial M$. By [Sc, Theorem 2.5], there is a finite covering $q: X\left(M^{\prime}\right) \rightarrow X(M)$ of orbifold such that $X\left(M^{\prime}\right)$ is an orientable surface. The pull-back of the Seifert fibration $p: M \rightarrow X(M)$ via $q$ gives a covering manifold $q^{\prime}: M^{\prime} \rightarrow M$ with Seifert fibration $p^{\prime}: M^{\prime} \rightarrow$ $X\left(M^{\prime}\right)$. Since $X\left(M^{\prime}\right)$ is an orientable surface, the fibration $p^{\prime}$ is an orientable circle bundle over the orientable surface $F=X\left(M^{\prime}\right)$. By the remark before Lemma 5.3, after passing to a further finite covering if necessary, we may assume that $q^{\prime}$ is characteristic.

Let $f^{\prime}: M^{\prime} \rightarrow M^{\prime}$ be a lifting of $f$ which fixes a point $A^{\prime}$ with $q^{\prime}\left(A^{\prime}\right)=A$. By Lemma $5.3, A^{\prime}$ is $f^{\prime}$-related to some component $B^{\prime}$ of $\left(q^{\prime}\right)^{-1}(B)$, by a path $\gamma^{\prime}$ which is a lifting of $\gamma$. Since $A$ is a fixed point of $f$, by the above assumption $f$ reverses the orientation of the fiber containing $A$. As a lifting of $f$, the map $f^{\prime}$ reverses the orientation of the fiber containing $A^{\prime}$, and hence the orientation of all fibers because the fibers of $M^{\prime}$ can be coherently oriented. Since the orbifold homeomorphism $\widehat{f}^{\prime}: X\left(M^{\prime}\right) \rightarrow X\left(M^{\prime}\right)$ is a lifting of the (periodic, Anosov or pseudo-Anosov) map $\widehat{f}$, it is also periodic, Anosov or pseudo-Anosov. By Lemma $6.2, f^{\prime}$ has the FR-property. Hence there is a homotopy $h_{t}^{\prime}$ deforming $\gamma^{\prime}$ to $\beta^{\prime} \subset F i x\left(f^{\prime}\right)$ rel $\left(A^{\prime}, B^{\prime}\right)$. The projection of $h_{t}^{\prime}$ on $M$ is a homotopy $q^{\prime} \circ h_{t}^{\prime}: \gamma \sim q^{\prime} \circ \beta^{\prime}$ rel $(A, B)$. Since $q^{\prime} \circ \beta^{\prime}$ is a path in $\operatorname{Fix}(f)$, this proves that $f$ has the FR-property.

Suppose $\widehat{f}$ is Anosov or pseudo-Anosov. Then Fix $(\widehat{f})$, hence Fix $(f)$, are isolated points. For each point $x \in \mathrm{Fix}(f)$, choose a product neighborhood $N(x)=D \times I$, with each $p \times I$ in a Seifert fiber of $M$. By a fiberwise isotopy we may assume that $f$ is a product $f_{1} \times f_{2}$ in a smaller neighborhood $N_{1}(x)=D_{1} \times I$, where $f_{2}$ is the involution on $I$. The map $f_{1}: D \rightarrow D$ is a lifting of $\left.\widehat{f}\right|_{p\left(D_{1}\right)}$, which is of pseudo-Anosov type. Therefore $f$ is a type 1 standard map.

Now suppose $\widehat{f}$ is periodic. Then Fix $(\widehat{f})$ consists of geodesics, so Fix $(f)$ is a 1-dimensional submanifold. Let $C$ be a component of Fix $(f)$. Let $N(C)$ be a regular neighborhood of $C$ which is an $I$-bundle induced by the Seifert fibration, over a surface $A$, which is either an annulus, a Möbius band, or a rectangle. Let $q: N(C) \rightarrow A$ be the quotient map pinching each $I$-fiber to a point. The homeomorphism $f$ induces a map $f_{1}: A_{1} \rightarrow A$, where $A_{1}$ is a subsurface of $A$ containing $q(C)$. Notice that $p: M \rightarrow X(M)$ induces 
a map $r: A \rightarrow X(M)$, which is locally a covering map of orbifolds, and $f_{1}$ covers the orbifold homeomorphism $\widehat{f}$, i.e, $r \circ f_{1}=\widehat{f} \circ r$. Since $\widehat{f}$ is periodic with a 1-dimensional fixed point set, it is an involution, hence $f_{1}$ is locally an involution near each fixed point. Therefore, we may choose a smaller $A$ if necessary, so that $f_{1}$ is an involution on $A$. It is now clear that $f_{1}$ preserves some normal structure of the curve $q(C)$ on $A$, which then lifts to a normal structure on $N(C)$ preserved by $f$. This completes the proof that $f$ is a type 2 standard map.

The following result supplements Proposition 6.3. It covers fiber preserving homeomorphisms on manifolds which have spherical orbifolds.

Proposition 6.4. Suppose that $M$ is a closed Seifert fiber space with orbifold $X(M)$ a sphere with at most three cone points, or a projective plane $P$ with at most two cone points. Let $f: M \rightarrow M$ be a fiber-preserving orientation-preserving homeomorphism. Then $f$ is isotopic to a fixed point free homeomorphism.

Proof. By a fiber-preserving isotopy we may assume that $\widehat{f}: X(M) \rightarrow X(M)$ is periodic. This is obvious when $X(M)$ is a sphere with at most three cone points or a projective plane with at most one cone point, and follows from Lemma 2.3 when $X(M)=P(r, s)$. By roposition $6.3, f$ is isotopic to a homeomorphism such that Fix $(f)$ is a 1-manifold, and $f$ preserves a normal structure of Fix $(f)$. By Lemma 5.5 we can modify $f$ by an isotopy to remove all such fixed points.

\section{Homeomorphisms on torus bundles.}

A 1-orbifold $Y$ is an interval with 0,1 or 2 cone points at its ends, or an $S^{1}$. In the first case a torus bundle over $Y$ is a $T \times I$, in the second case a twisted $I$-bundle over the Klein bottle, in the third case a union of two twisted $I$ bundles over Klein bottle, and in the fourth case it is a torus bundle over $S^{1}$. In this section we will study homeomorphisms on these manifolds with each fiber a torus. In particular, we will prove Theorem 9.1 for homeomorphisms on Sol manifolds.

Lemma 7.1. If $M$ is a twisted I-bundle over the Klein bottle, then an orientation-preserving homeomorphism $f: M \rightarrow M$ can be deformed by an isotopy to a type 2 standard map. 
Proof. According to [WW, Lemma 1.1], up to isotopy $M$ admits exactly two Seifert fibrations: It is a twisted circle bundle over Möbius band, and it is also a Seifert fiber space over a disk with two singular points of index 2 . Since those two Seifert fibrations are not homeomorphic, the homeomorphism $f$ must preserve each Seifert fibration up to isotopy. So we may assume that $f$ is a fiber preserving homeomorphism. By Proposition 2.4, with a further isotopy we may assume that the orbifold map $\widehat{f}$ is periodic. Hence we can apply Proposition 6.3 to modify $f$ by an isotopy to a type 2 standard map.

Lemma 7.2. Let $N=T \times I$, where $T$ is a torus. Let $T_{i}=T \times i$, and let $f_{i}=\left.f\right|_{T_{i}}$. Suppose $f: N \rightarrow N$ is an orientation-preserving homeomorphism which is standard on the boundary.

(1) If $f_{i}\left(T_{i}\right) \neq T_{i}$, then $f$ is rel $\partial N$ isotopic to a type 1 standard map $g$. Moreover, if $f_{i}^{2}: T_{i} \rightarrow T_{i}$ is not isotopic to an Anosov map, then $g$ is fixed point free.

(2) If $f_{i}\left(T_{i}\right)=T_{i}$, then $f$ is rel $\partial N$ isotopic to a type 2 standard map.

Proof. (1) Suppose $f_{i}\left(T_{i}\right) \neq T_{i}$. We may assume that $f$ maps $T=T \times \frac{1}{2}$ to itself. By Lemma 5.12 we may modify $f^{\prime}=\left.f\right|_{T}$ by an isotopy to a fixed point free or Anosov map. The isotopy can be extended to an isotopy of $f$ rel $\partial N$, modifying $f$ to a homeomorphism $g$ so that in a neighborhood of $T$ it is the product of $f^{\prime}$ with an involution. Each point of Fix $(g)$ is an essential fixed point class of $g$, and since $g$ has no invariant boundary component, no point of Fix $(g)$ is $g$-related to a boundary component, so $g$ has the FR-property. Therefore $g$ is a type 1 standard map, and $g$ is fixed point free unless $f^{\prime}$ is Anosov, which is true if and only if $f_{i}^{2}: T_{i} \rightarrow T_{i}$ is isotopic to an Anosov map.

(2) Suppose $f_{i}\left(T_{i}\right)=T_{i}$. We separate the argument into several cases according to the type of $f_{i}$ in Definition 5.8. Note that the three types of $f_{i}$ are mutually exclusive up to isotopy, hence $f_{0}$ and $f_{1}$ have the same type.

Case 1. $f_{i}$ has no fixed point.

In this case we can just modify $f$ by an isotopy rel $\partial N$ so that for any $x \in(0,1), f$ sends the torus $T \times x$ to $T \times y$ for some $y \in(0, x)$. Then $f$ has no fixed point on $N(T)$, so it is automatically a type 2 standard map.

Case 2. $f_{i}$ is a periodic map with isolated fixed points. 
In this case it follows from [JW, Proposition 3.1] that $f$ is rel $\partial N$ isotopic to an affine map $g$ with respect to some Euclidean structure of $T \times I$, so that each component of Fix $(g)$ is an arc from $T_{0}$ to $T_{1}$. (Note that since $\left.f\right|_{T_{i}}$ is orientation-preserving and is not the identity map, Fix $(f)$ can not be torus or circle on $T_{i}$, which rules out the other possibilities of [JW, Prop. 3.1].) Since $g$ is affine, it maps each $T \times t$ to itself, hence it preserves a normal structure.

Every path class in $T \times I$ contains a unique straight path. Since $g$ is an affine map, any straight path connecting fixed points is pointwise fixed by $g$. Hence $g$ has the FR-property.

Case 3. $f_{i}$ is a fiber-preserving, fiber-orientation-reversing homeomorphism with respect to some $S^{1}$ fibration of $T_{i}$.

In this case the fiber direction of $f_{i}$ is uniquely determined by the isotopy type of $f_{i}$, hence the two fibrations on $T_{i}$ are isotopic to each other. The fibrations on $T_{i}$ extends to an $S^{1}$-bundle structure of $N$ over an annulus $A$, and $f$ is rel $\partial N$ isotopic to a fiber-preserving map. The induced homeomorphism $\hat{f}: A \rightarrow A$ is orientation-reversing, so it is rel $\partial A$ isotopic to an involution. The result now follows immediately from Lemma 6.2.

Recall that $N(f)$ denotes the Nielsen number of a map $f$.

Lemma 7.3. Suppose $M$ is a torus bundle over $S^{1}$, and $f: M \rightarrow M$ is an orientation-preserving homeomorphism which preserves the torus fibration. Then $f$ can be deformed by an isotopy to a homeomorphism $g$ with exactly $N(f)$ fixed points. Furthermore, $N(f)=0$ unless (i) the induced homeomorphism $f^{\prime}: S^{1} \rightarrow S^{1}$ is orientation-reversing, and (ii) the restriction of $f$ on at least one of the invariant torus fibers is isotopic to an Anosov map.

Proof. If $f^{\prime}: S^{1} \rightarrow S^{1}$ is orientation-preserving, then we can deform $f$ via a fiber-preserving isotopy, so that $f^{\prime}$ is fixed point free, hence $f$ is also fixed point free, and the result follows.

If $f^{\prime}$ is orientation-reversing, then $f$ has two invariant torus fibers $T_{1}$ and $T_{2}$, and $F i x(f) \subset T_{1} \cup T_{2}$. By the Nielsen Realization Theorem of surface homeomorphisms [JG, Main Theorem], we may deform $f_{i}=\left.f\right|_{T_{i}}$ by an isotopy so that $N\left(f_{i}\right)=\#$ Fix $\left(f_{i}\right)$ as homeomorphisms on tori. The isotopy can be extended to a fiber-preserving isotopy of $f$, deforming $f$ to a new homeomorphism $g$ such that the restriction of $g$ on $N\left(T_{i}\right)=T_{i} \times I$ is a product $f_{i} \times \varphi$, where $\varphi: I \rightarrow I$ is the involution $\varphi(t)=1-t$. Since $T_{i}$ are still the only fibers preserved by $g$, we have Fix $(g)=F i x\left(f_{1}\right) \cup \operatorname{Fix}\left(f_{2}\right)$. 
Let $t=1 / 2$ be the fixed point of $\varphi$. Note that since $\varphi$ is an involution, the index of $t$ is 1 . Since $g$ is locally a product $f_{i} \times \varphi$ near each fixed point, by Lemma 5.2 we have

$$
\text { ind }(f, x)=\operatorname{ind}\left(f_{i}, x\right) \times \operatorname{ind}(\varphi, t)
$$

for any $x \in \operatorname{Fix}(f)$. It follows that the index of each fixed point of $g$ is nonzero. We need to show that no two of them are Nielsen equivalent.

Suppose $x, y \in \operatorname{Fix}(g)$ are in the the same fixed point class of $g$. Then there is a path $\gamma$ from $x$ to $y$ such that

$$
g \circ \gamma \sim \gamma \quad \text { rel } \partial
$$

Let $\gamma^{\prime}=q \circ \gamma$. Then $\gamma^{\prime}$ is a path from $q(x)$ to $q(y)$, and

$$
g^{\prime} \circ \gamma^{\prime} \sim \gamma^{\prime} \quad \text { rel } \partial
$$

where $g^{\prime}$ is the orbifold map on $S^{1}$ induced by $g$. Since $g^{\prime}$ is orientationreversing, this implies that $\gamma^{\prime}$ is homotopic to a trivial loop. Thus $x$ and $y$ must be in the same $T_{i}$, and $\gamma$ can be deformed into $T_{i}$. Now $\pi_{1}\left(T_{i}\right)$ injects into $\pi_{1}(M)$, so the homotopy $\gamma \sim f \circ \gamma$ can be deformed into $T_{i}$, which implies that $x$ and $y$ are in the same fixed point class of $f_{i}$, contradicting the fact that \#Fix $\left(f_{i}\right)=N\left(f_{i}\right)$. Therefore the number of fixed points of $g$ is exactly $N(f)$.

By Lemma 5.12, $f_{i}$ is isotopic to a fixed point free or Anosov map. So if neither $f_{i}$ is isotopic to an Anosov map, then $g$ is fixed point free.

Denote by $J$ the 1 -orbifold $[-1,1]$ with two singular points at its ends. If $p: M \rightarrow J$ is a torus fibration, then $M$ is a union of two twisted $I$-bundle over the Klein bottle, glued together along their boundary.

Lemma 7.4. Suppose $M$ is a torus bundle over the 1-orbifold $J$, and $f$ : $M \rightarrow M$ is an orientation-preserving homeomorphism which preserves the torus fibration. Then $f$ is isotopic to a homeomorphism with no fixed points.

Proof. Let $f^{\prime}: J \rightarrow J$ be the orbifold map induced by $f$. By an isotopy we may assume that $f^{\prime}$ is either the identity or an involution on $J$. In particular, $f^{\prime}$ fixes the middle point of $J$, so the middle torus fiber $T$ is invariant under the homeomorphism $f$.

First assume that $f^{\prime}$ is an involution on $J$. As in the proof of Lemma 7.3, $\left.f\right|_{T}$ is isotopic to either a fixed point free homeomorphism, or an Anosov 
map. Now $T$ cuts $M$ into $N_{1}, N_{2}$, which are twisted $I$-bundles over the Klein bottle. The homeomorphism $f^{2}$ sends each $N_{i}$ to itself, so by Proposition 2.4 it is isotopic to a periodic map. In particular, $\left.f^{2}\right|_{\partial N_{i}}=\left(\left.f\right|_{T}\right)^{2}$ is isotopic to a periodic map, so $\left.f\right|_{T}$ can not be an Anosov map. Thus $\left.f\right|_{T}$ is isotopic to a fixed point free homeomorphism. The isotopy can be fiberwise extended over $M$, modifying $f$ to a fixed point free homeomorphism.

Now assume that $f^{\prime}: J \rightarrow J$ is the identity map. Since $N_{i}$ is a twisted $I$-bundle over the Klein bottle, by Lemma 7.1 we can deform $f$ by an isotopy so that $\left.f\right|_{N_{i}}$ is a Type 2 standard map. By Lemma 7.2 we can deform $\left.f\right|_{N(T)}$ by an isotopy rel $\partial N(T)$ to a Type 2 standard map. Thus Fix $(f)$ is a union of 1-manifold in $N_{i}$ and $N(T)$, with some normal structure preserved by $f$. Since $\left.f\right|_{N_{i}}$ and $\left.f\right|_{N(T)}$ have no isolated fixed points, Fix $(f)$ must be a union of circles in $M$. By Lemma 5.5 one can modify $f$ by an isotopy to eliminate all such fixed points, and get a fixed point free homeomorphism.

Proposition 7.5. Suppose $M$ is an orientable 3-manifold with Sol geometry and $f: M \rightarrow M$ is an orientation-preserving homeomorphism. Then $f$ is isotopic to a fixed point free homeomorphism.

Proof. A Sol manifold has a structure of torus bundle over 1-orbifold, and by [Sc, Theorem 5.3] it is not a Seifert fiber space. Thus by Lemmas 3.7 and 3.8, we may assume that $f$ is torus fiber-preserving. If $M$ is a torus bundle over the 1-orbifold $J$, the result follows from Lemma 7.4. Hence we may assume that $M$ is a torus bundle over $S^{1}$. By Lemma 7.3, $f$ can be deformed by an isotopy so that \#Fix $(f)=N(f)$. It remains to show that $N(f)=0$.

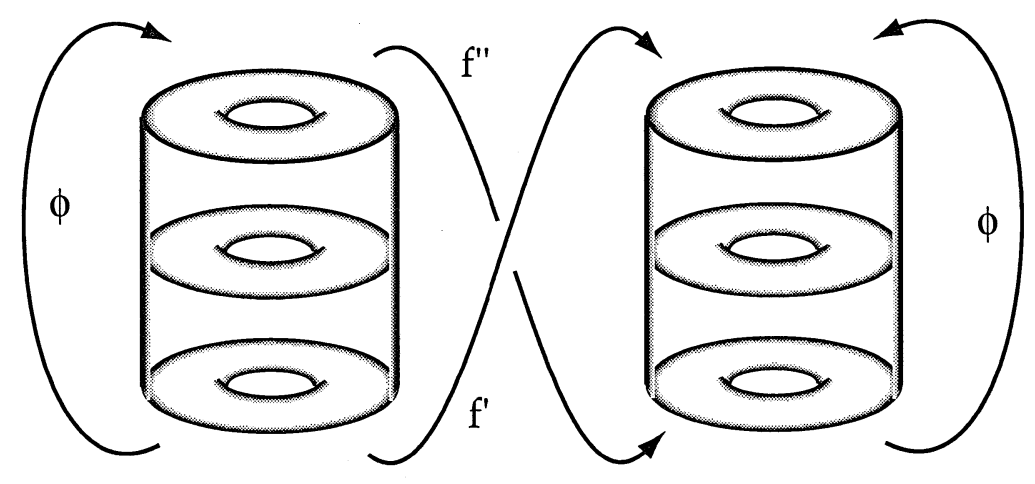

Figure 7.1 
Continue with the proof of Lemma 7.3. We have shown that $f$ induces an involution on the orbifold $S^{1}$, whose two fixed points yield two $f$-invariant tori $T_{1}, T_{2}$ of $M$, and $\operatorname{Fix}(f)=\operatorname{Fix}\left(f_{1}\right) \cup \operatorname{Fix}\left(f_{2}\right)$, where $f_{i}=\left.f\right|_{T_{i}}$. We may consider $M$ as a quotient

$$
M=(T \times I) / \varphi
$$

where

$$
\varphi: T \times 0 \rightarrow T \times 1
$$

is the gluing map. We may assume that $T_{1}=T \times 1=T \times 0$, and $T_{2}=T \times \frac{1}{2}$. Thus $f$ pulls back to a torus fiber-preserving homeomorphism

$$
\widehat{f}: T \times I \rightarrow T \times I
$$

which induces an involution on $I$. Put $f^{\prime}=\left.\widehat{f}\right|_{T \times 0}$, and $f^{\prime \prime}=\left.\widehat{f}\right|_{T \times 1}$. See Figure 7.1. We have the following commutative diagram.

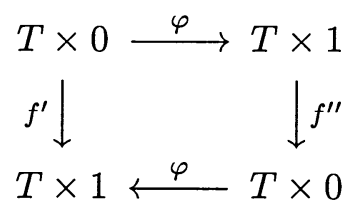

Fixing a basis for $H_{1}(T)$, each map then determines a matrix with respect to this basis. Let $A$ be the matrix of $\varphi$. Since $f^{\prime}$ and $f^{\prime \prime}$ are the restriction of $\widehat{f}$, they induce the same matrix $B$. The above commutative diagram implies that

$$
B A^{-1}=A B
$$

Since $M$ is a Sol manifold, the gluing map $\varphi$ is an Anosov map, so the matrix $A$ has two distinct real eigenvalues $\lambda_{1}, \lambda_{2}$. Let $v_{i}$ be an eigenvector of $\lambda_{i}$. With respect to the basis $\left(v_{1}, v_{2}\right)$ the map $\varphi$ is represented by a diagonal matrix with diagonal entries $\lambda_{1}$ and $\lambda_{2}$. Let $B^{\prime}$ be the matrix representing $f^{\prime}$ and $f^{\prime \prime}$ with respect to this new basis. Then

$$
B^{\prime}\left(\begin{array}{cc}
\lambda_{2} & 0 \\
0 & \lambda_{1}
\end{array}\right)=\left(\begin{array}{cc}
\lambda_{1} & 0 \\
0 & \lambda_{2}
\end{array}\right) B^{\prime} .
$$

Since $\lambda_{1} \neq \lambda_{2}$, by comparing the coefficient in the above equation we have

$$
B^{\prime}=\left(\begin{array}{cc}
0 & b_{12} \\
b_{21} & 0
\end{array}\right),
$$

hence $\operatorname{tr}(B)=\operatorname{tr}\left(B^{\prime}\right)=0$. 
Denote by $h$ the map $f_{2}: T_{2} \rightarrow T_{2}$. Let

$$
h_{i *}: H_{i}\left(T_{2}\right) \rightarrow H_{i}\left(T_{2}\right)
$$

be the induced homomorphism on the $i$-th homology group of $T_{2}$. The matrix $B$ is also the representation matrix of $h_{1 *}$. According to [Ki], the Nielsen number of a torus map is the same as the absolute value of its Lefschetz number:

$$
N(h)=\left|\operatorname{tr}\left(h_{0 *}\right)-\operatorname{tr}\left(h_{1 *}\right)+\operatorname{tr}\left(h_{2 *}\right)\right| .
$$

Clearly $\operatorname{tr}\left(h_{0 *}\right)=1$. Since $h=f_{2}$ is orientation-reversing on $T_{2}$, we have $\operatorname{tr}\left(h_{2 *}\right)=-1$. Therefore

$$
N\left(f_{2}\right)=N(h)=\operatorname{tr}\left(h_{1 *}\right)=\operatorname{tr}(B)=0 .
$$

Similarly, one can show that $N\left(f_{1}\right)=0$. Since $\operatorname{Fix}(f)=\operatorname{Fix}\left(f_{1}\right) \cup$ Fix $\left(f_{2}\right)$, this implies that $N(f)=0$.

\section{Homeomorphisms on some geometric manifolds.}

There are eight 3-manifold geometries, see [Sc, Theorem 5.3]. In this section we will prove the main theorem for six types of geometric manifolds. The remaining two geometries are the $H^{2} \times \mathbb{R}$ and $\widetilde{S L_{2} \mathbb{R}}$. These are Seifert fibred manifolds with hyperbolic orbifolds, and will be treated in Section 9, together with manifolds admitting nontrivial JSJ splittings.

If $M$ is a manifold with toroidal boundary whose interior admits a complete hyperbolic structure, then we can identify $M$ with the $\epsilon$-thick part of a complete hyperbolic manifold. More explicitly, the interior $X$ of $M$ admits a complete hyperbolic structure with finite volume. Let $X_{\epsilon}$ be the set of all points $x$ in Int $X$ such that there is an embedded hyperbolic open ball of radius $\epsilon$ centered at $x$. There exists an $\epsilon_{0}>0$ such that for any $\epsilon \leq \epsilon_{0}, X_{\epsilon}$ is homeomorphic to $M$ and each boundary component of $X_{\epsilon}$ is a horosphere modulo a rank two abelian group of parabolic motions. (See [Th1, 5.11].) We identify $M$ with such an $X_{\epsilon}$.

Lemma 8.1. Let $f$ be an orientation-preserving homeomorphism of an orientable hyperbolic manifold $M$. Then $f$ is isotopic to a type 2 standard map $g$.

Proof. Since $M$ is identified with $X_{\epsilon}$, the homeomorphism $f: M \rightarrow M$ extends to a homeomorphism $f^{\prime}: X \rightarrow X$. By Mostow's Rigidity Theorem 
[Mo, p. 54], there is a unique isometry $g^{\prime}: X \rightarrow X$ which is homotopic to $f^{\prime}$. Since $X$ retracts to $X_{\epsilon}=M$, the homeomorphism $g=\left.g^{\prime}\right|_{M}$ is homotopic to $f$. By a recent result of Gabai, Meyerhoff and Thurston [Ga, GMT], $f$ is actually isotopic to the isometry $g: M \rightarrow M$.

If $g=i d$, then by a small flow along a non vanishing vector field which is tangential on the boundary, we get a fixed point free homeomorphism, and we are done. So assume that $g \neq i d$.

Suppose each of $A_{0}, A_{1}$ is either a fixed point of $g$ or a boundary component of $M$, and $A_{0}$ is $g$-related to $A_{1}$ via the path $\gamma$. The universal covering $\widetilde{M}$ of $M$ is the hyperbolic 3-space $H^{3}$ with some horospheres removed. By lifting $\gamma$ to $\widetilde{M}$ we see that $\gamma$ is $\left(A_{0}, A_{1}\right)$ homotopic to a geodesic $\gamma^{\prime}$, which is perpendicular to $A_{i}$ if it is a boundary component. Moreover, such a geodesic $\gamma^{\prime}$ is unique among all paths which are $\left(A_{0}, A_{1}\right)$ homotopic to $\gamma$. Since $g$ is an isometry, the path $g \circ \gamma^{\prime}$ is also a geodesic, and

$$
g \circ \gamma^{\prime} \sim g \circ \gamma \sim \gamma \sim \gamma^{\prime} \quad \operatorname{rel}\left(A_{0}, A_{1}\right) .
$$

So by the uniqueness of $\gamma^{\prime}$, we must have $\gamma^{\prime}=g \circ \gamma^{\prime}$. In particular, $\gamma^{\prime}$ is a path in Fix $(g)$. Therefore $g$ has the FR-property. Since $g$ is a non identity orientation-preserving isometry, $\operatorname{Fix}(g)$ consists of mutually disjoint geodesics, so it is a properly embedded 1-manifold. A neighborhood of Fix $(g)$ is parametrized by a family of hyperbolic disks perpendicular to Fix $(g)$, each of which is preserved by the isometry $g$, hence $g$ preserved a normal structure of Fix $(g)$. It is known that isometries on hyperbolic manifolds are periodic [Th1], so in this case $\left.g\right|_{\partial M}$ is a periodic map with isolated fixed points.

Corollary 8.2. If $M$ is a closed hyperbolic manifold, and $f: M \rightarrow M$ is an orientation-preserving homeomorphism, then $f$ is isotopic to a fixed point free homeomorphism.

Proof. By Lemma 8.1 we may assume that $f$ is a type 2 standard map, so Fix $(f)$ is a 1-manifold. Since $M$ is closed, Fix $(f)$ consists of circles, which, by Lemma 5.5 , can be removed via an isotopy.

Lemma 8.3. Let $f$ be a homeomorphism on the manifold $T^{3}$. Then $f$ is isotopic to a homeomorphism $g$ with \#Fix $(g)=N(f)$.

Proof. Identify $T^{3}$ with $\mathbb{R}^{3} / \mathbb{Z}^{3}$, and identify the fundamental group $\pi_{1}\left(T^{3}\right)$ with the covering translation group $\mathbb{Z}^{3}$. The homeomorphism $f: T^{3} \rightarrow T^{3}$ 
induces an automorphism $f_{*}: \mathbb{Z}^{3} \rightarrow \mathbb{Z}^{3}$. The latter extends to a linear automorphism $\tilde{g}: \mathbb{R}^{3} \rightarrow \mathbb{R}^{3}$, inducing a linear automorphism $g: T^{3} \rightarrow T^{3}$ which is homotopic to $f$.

Let $A$ be the matrix of $f_{*}$, and $E$ be the identity matrix. According to [J1, Example 2 of $\S I I .4$ and I.3.2(2)], if $A$ does not have 1 as an eigenvalue, then the linear homeomorphism $g$ has exactly $N(f)=|\operatorname{det}(E-A)|$ fixed points, all having the same index which equals the sign of $\operatorname{det}(E-A)$. If 1 is an eigenvalue, then $N(f)=0$, and an arbitrarily small translation in the direction of a 1-eigenvector removes all fixed points. Thus we obtain an affine homeomorphism, still denoted by $g$, with \#Fix $(g)=N(f)$, which is homotopic to $f$.

Since $T^{3}$ is a Haken manifold, $g$ is in fact isotopic to $f$.

Lemma 8.4. If $f$ is an orientation-preserving homeomorphism on the manifold $M=M_{P(2,2)}$, then it is isotopic to a fixed point free homeomorphism.

Proof. By Theorem $4.1 f$ is isotopic to an isometry with respect to some Euclidean metric of $M$. Since $f$ is orientation-preserving, its fixed point set consists of disjoint circles, which can be removed by Lemma 5.5.

Proposition 8.5. If $M$ is a closed orientable 3-manifold which admits a Sol, Nil, $H^{3}, E^{3}, S^{3}$ or $S^{2} \times \mathbb{R}$ geometry, then any orientation-preserving homeomorphism $f$ on $M$ is isotopic to a homeomorphism $g$ such that \#Fix $(g)=N(f)$.

Proof. If $M$ admits a Sol or hyperbolic manifold, $T^{3}$, or $M_{P(2,2)}$, the result follows from Proposition 7.5, Lemmas 8.2, 8.3 and 8.4, respectively. So assume that $M$ is not such a manifold. All the remaining manifolds are Seifert fiber spaces. By Theorem 3.11 the map $f$ is isotopic to a fiber-preserving homeomorphism with respect to some Seifert fibration of $M$ which has spherical or Euclidean orbifold. If $X(M)$ is Euclidean, by Theorem 2.5 the induced orbifold map $\widehat{f}$ is isotopic to a periodic, reducible, or Anosov map. Note that this is trivially true if $X(M)$ is spherical because all homeomorphisms on a spherical orbifold are isotopic to periodic maps.

If $\widehat{f}$ is Anosov, by Proposition $6.3 f$ is isotopic to a Type 1 map, and by Lemma $5.7 f$ can be further deformed by an isotopy so that $N(f)=$ \#Fix $(f)$, as desired. If $X(M)$ is spherical, by Proposition $6.4 f$ is isotopic to a fixed point free homeomorphism. If $\widehat{f}$ is periodic and $X(M)$ is Euclidean, by Proposition $6.3 f$ is isotopic to a Type 2 standard map $g$. Since $M$ 
is closed, each component of Fix $(g)$ is a circle; hence by Lemma $5.5 \mathrm{~g}$ is isotopic to a fixed point free homeomorphism.

Now assume that $\widehat{f}$ is reducible, and let $C$ be a reducing curve on $X(M)$. Then $M$ also has the structure of a torus bundle over a 1-orbifold, with the torus $T$ over $C$ a torus fiber. Since $f(T)=T$, we can deform $f$ by an isotopy to a torus fiber-preserving map. The Seifert fibration provides an $S^{1}$ bundle structure on $T$, which is preserved by $f$, hence $\left.f\right|_{T}$ is a reducible homeomorphism. We can now apply Lemmas 7.3 and 7.4 to conclude that $f$ is isotopic to a homeomorphism with no fixed points.

\section{Proof of the main theorems.}

Theorem 9.1. Suppose $M$ is a closed orientable 3-manifold which is either Haken or geometric, and $f: M \rightarrow M$ is an orientation-preserving homeomorphism. Then $f$ can be deformed by an isotopy to a homeomorphism $g$ with \#Fix $(g)=N(f)$.

Proof. If $M$ admits a Sol, Nil, $E^{3}, S^{3}$ or $S^{2} \times \mathbb{R}$ geometry, the result follows from Proposition 8.5. Assume that $M$ is not such a manifold, and let $\mathcal{T}$ be the (possibly empty) minimal set of tori of the Jaco-Shalen-Johannson decomposition. Then each component of $M-\operatorname{Int} N(\mathcal{T})$ is either hyperbolic, or a twisted $I$-bundle over Klein bottle, or a Seifert fiber space with hyperbolic orbifold. By an isotopy we can deform $f$ so that it maps $N(\mathcal{T})$ homeomorphically to itself.

Suppose that $P$ is a Seifert fibered component of $M-\operatorname{Int} N(\mathcal{T})$ such that $f(P)=P$. By [Sc, Theorem 3.9], $\left.f\right|_{P}$ is isotopic to a fiber-preserving map. By Lemma 1.10 and Proposition 2.4, we can find a set of vertical tori $\mathcal{T}_{P}$ in $P$, cutting $P$ into pieces with hyperbolic or $D(2,2)$ orbifold, and a fiber-preserving isotopy of $f$, so that after isotopy, the restriction of $f$ on each invariant piece has periodic or pseudo-Anosov orbifold map. Adding all such $\mathcal{T}_{P}$ to $\mathcal{T}$, we get a collection of tori $\mathcal{T}^{\prime}$, such that

(i) $f\left(N\left(\mathcal{T}^{\prime}\right)\right)=N\left(\mathcal{T}^{\prime}\right)$;

(ii) Each component $M_{i}$ of $M-\operatorname{Int} N\left(\mathcal{T}^{\prime}\right)$ is either hyperbolic, or a Seifert fiber space such that $X\left(M_{i}\right)$ is a hyperbolic orbifold or $D(2,2)$.

(iii) If $f$ maps a Seifert fibered component $M_{i}$ to itself, and if $M_{i}$ has hyperbolic orbifold, then $f$ is fiber-preserving, and the orbifold map $\widehat{f}$ on $X\left(M_{i}\right)$ is either periodic or pseudo-Anosov. 
We can now apply Proposition 6.3, Lemma 7.1 and Lemma 8.1 to each component $M_{i}$ of $M-\operatorname{Int} N\left(\mathcal{T}^{\prime}\right)$ which is mapped to itself by $f$, isotoping $f$ so that $\left.f\right|_{M_{i}}$ is a standard map for all $M_{i}$. After that, use Lemma 7.2 to further deform $f$ by an isotopy rel $\partial N_{j}$ on each component $N_{j}$ of $N\left(\mathcal{T}^{\prime}\right)$, so that it is a standard map on $N_{j}$.

By the definition of standard maps, Fix $(f)$ intersects each of $M_{i}$ and $N_{j}$ in isolated points and properly embedded 1-manifolds, so Fix $(f)$ is a disjoint union of points, arcs, and circles. Moreover, at each isolated point of Fix $(f)$ the homeomorphism $f$ is of flipped-pseudo-Anosov type, and at each arc or circle it preserves some normal structure.

We want to show that different components of Fix $(f)$ are not equivalent. If this is not true, there is a path $\alpha$ connecting different components $C_{0}, C_{1}$ of Fix $(f)$, such that $f \circ \alpha \sim \alpha$ rel $\partial$. Denote by $\mathcal{T}^{\prime \prime}$ the set of tori $\partial N\left(\mathcal{T}^{\prime}\right)$. Among all such $\alpha$, choose one such that $\left|\alpha-\mathcal{T}^{\prime \prime}\right|$ is minimal. If $\left|\alpha-\mathcal{T}^{\prime \prime}\right|=0$ or 1 , then $\alpha$ lies in some $M_{i}$ or $N_{j}$, which we denote by $X$. Since $\alpha$ contains some fixed point of $f, f$ must map $X$ to itself. Since $X$ is a component of $M$ cut along incompressible tori, $\pi_{1}(X)$ maps injectively into $\pi_{1}(M)$; so $\alpha$ and $f \circ \alpha$ being rel $\partial$ homotopic in $M$ implies that they are rel $\partial$ homotopic in $X$. But since $f$ is a standard map on $X$, the two endpoints of $\alpha$ belong to the same component of $\operatorname{Fix}\left(\left.f\right|_{X}\right)$, which is a contradiction. Hence we may assume that $\left|\alpha-\mathcal{T}^{\prime \prime}\right| \geq 2$. Below, we will find another such curve $\alpha^{\prime \prime}$ with $\left|\alpha^{\prime \prime}-\mathcal{T}^{\prime \prime}\right|<\left|\alpha-\mathcal{T}^{\prime \prime}\right|$, which would contradict the choice of $\alpha$.

Let $D$ be a disk, and let $h: D \rightarrow M$ be the homotopy $\alpha \sim f \circ \alpha$ rel $\partial$. We may assume that $h$ is transverse to $\mathcal{T}^{\prime \prime}$, and $\left|h^{-1}\left(\mathcal{T}^{\prime \prime}\right)\right|$ is minimal among all such $h$. Then $h^{-1}\left(\mathcal{T}^{\prime \prime}\right)$ consists of a properly embedded 1-manifold on $D$, together with possibly one or two isolated points mapped to the ends of $\alpha$. Clearly, $\mathcal{T}^{\prime \prime}$ is $\pi_{1}$-injective in $M$, so one can modify $h$ to remove all circles in $h^{-1}\left(\mathcal{T}^{\prime \prime}\right)$. Note that $h^{-1}\left(\mathcal{T}^{\prime \prime}\right)$ must contain some arcs because $\left|\alpha-\mathcal{T}^{\prime \prime}\right| \geq 2$.

Now consider an outermost arc $b$ in $h^{-1}\left(\mathcal{T}^{\prime \prime}\right)$. Let $\beta=h(b)$. The ends of $\beta$ can not both be on $\alpha$, otherwise we can use the outermost disk to homotope $\alpha$ and reduce $\left|\alpha-\mathcal{T}^{\prime \prime}\right|$, contradicting the choice of $\alpha$. Since $f$ is a homeomorphism preserving $\mathcal{T}^{\prime \prime}$, the same thing is true for $f \circ \alpha$. Therefore, $\beta$ has one end on each of $\alpha$ and $f \circ \alpha$.

The arc $b$ cuts off a disk $\Delta$ on $D$ whose interior is disjoint from $h^{-1}\left(\mathcal{T}^{\prime \prime}\right)$. The boundary of $\Delta$ gives rise to a loop

$$
h(\partial \Delta)=\alpha_{1} \cup \beta \cup\left(f \circ \alpha_{1}\right)^{-1},
$$

where $\alpha_{1}$ is a subpath of $\alpha$ starting from an end point $x$ of $\alpha$. Let $T$ be the torus in $\mathcal{T}^{\prime \prime}$ which contains $\beta$. Then the restriction of $h$ on $\Delta$ gives a 
homotopy

$$
\alpha_{1} \sim f \circ \alpha_{1} \operatorname{rel}(x, T) .
$$

Since $f$ has the FR-property on each component of $M-\operatorname{Int} N(\mathcal{T})$ and $N(\mathcal{T})$, by definition there is a path $\gamma$ in Fix $(f)$ such that $\gamma \sim \alpha_{1}$ rel $(x, T)$. Since $\gamma$ is in Fix $(f)$, the path $\alpha^{\prime}=\gamma^{-1} \cdot \alpha$ has the property that

$$
f \circ \alpha^{\prime}=\left(f \circ \gamma^{-1}\right) \cdot(f \circ \alpha)=\gamma^{-1} \cdot(f \circ \alpha) \sim \gamma^{-1} \cdot \alpha=\alpha^{\prime} \quad \text { rel } \partial .
$$

Since $\alpha_{1} \sim \gamma \operatorname{rel}(x, T)$, the path $\gamma^{-1} \cdot \alpha_{1}$ is rel $\partial$ homotopic to a path $\delta$ on $T$. Write $\alpha=\alpha_{1} \cdot \alpha_{2}$. Then

$$
\alpha^{\prime}=\gamma^{-1} \cdot \alpha=\left(\gamma^{-1} \cdot \alpha_{1}\right) \cdot \alpha_{2} \sim \delta \cdot \alpha_{2} \text { rel } \partial
$$

By a small perturbation on $\delta$, we get a path $\alpha^{\prime \prime} \sim \alpha^{\prime}$ rel $\partial$ such that $\mid \alpha^{\prime \prime}-$ $\mathcal{T}^{\prime \prime}|<| \alpha-\mathcal{T}^{\prime \prime} \mid$. Since

$$
f \circ \alpha^{\prime \prime} \sim f \circ \alpha^{\prime} \sim \alpha^{\prime} \sim \alpha^{\prime \prime} \text { rel } \partial
$$

this contradicts the minimality of $\left|\alpha-\mathcal{T}^{\prime \prime}\right|$.

So far we have proved that each component of $F i x(f)$ is a Nielsen class of $f$. Our next goal is to show that we can deform $f$ by an isotopy in a neighborhood of each arc component $C$ of Fix $(f)$ so that the class $C$ becomes a single point, or is completely deleted if ind $(f, C)=0$.

Notice that $C$ is the union of several arcs $C_{k}$, each being a component of some Fix $\left(\left.f\right|_{M_{i}}\right)$ or Fix $\left(\left.f\right|_{N_{j}}\right)$, and the ends of $C$ are isolated points of, say, Fix $\left(\left.f\right|_{\partial M_{0}}\right)$ and Fix $\left(\left.f\right|_{\partial M_{1}}\right)$. Since $f$ is a standard map on each piece, we can identify $N(C)$ with a subset of $\mathbb{R}^{3}$ as follows:

(1) $N(C)=[-3,3] \times D$, where $D=[-2,2] \times[-2,2]$.

(2) $C=[-1,1] \times(0,0)$.

(3) For $t \in[-1,1], f$ maps $t \times D_{1}$ into $t \times D$, where $D_{1}=[-1,1] \times[-1,1]$ is a smaller neighborhood of $(0,0)$. This is because $f$ preserves a normal structure on each arc component of Fix $\left(\left.f\right|_{M_{i}}\right)$ or Fix $\left(\left.f\right|_{N_{j}}\right)$.

(4) On $[-2,-1] \times D$, the map has the property that $f(x, y, z)=$ $(\varphi(x, y),-z)$, where $\varphi(x, y)$ has the property that it switches the two sides of the $x y$-plane on the two sides of the $x$-axis, i.e, if $\varphi(x, y)=\left(x^{\prime}, y^{\prime}\right)$ then $y^{\prime}>0$ if and only if $y<0$. This follows from the fact that the point $(-1,0,0)$ is an isolated point of Fix $\left(\left.f\right|_{M_{0}}\right)$, hence is of flipped-pseudo-Anosov type. Similarly for $[1,2] \times D$. 
Consider the ball $B_{1}=[-2,2] \times D_{1}$. Define a map $h: B_{1} \rightarrow \mathbb{R}^{3}$ by $h(v)=v-f(v)$. It follows from the above discussion that $\left.f\right|_{B_{1}}$ have the following properties.

(5) $h(v)$ is parallel to the $x$ axis if and only $v=(x, 0,0)$ for some $x$. Moreover, for all $-2 \leq x<-1, h(v)$ points to the same direction of the $x$-axis. Similarly for all $1<x \leq 2$.

We can now compute ind $(f, C)$. Put $e=(1,0,0)$. By (5) there are only two points on $\partial B_{1}$ that are mapped to $\pm e$ by the map $\bar{h}(v)=h(v) /\|h(v)\|$, namely the points $\pm 2 e$. It follows that the degree of the map $\bar{h}: \partial B_{1} \rightarrow S^{2}$ is either 0 or \pm 1 , depending on whether $\bar{h}(2 e)=\bar{h}(-2 e)$ or not.

If $\bar{h}(2 e)=\bar{h}(-2 e)=e$, say, then $v-f(v)$ is never a positive multiple of $-e$. Define an isotopy supported in a small neighborhood of $C$ by flowing along the direction of $-e$. This isotopy will modify $f$ to a homeomorphism $g$ with no fixed point in $B_{1}$.

If $\bar{h}(2 e)=e$ and $\bar{h}(-2 e)=-e$, say, we can define an isotopy supported in a small neighborhood of $C$ by flowing each point $(x, y, z)$ towards $(0, y, z)$. This will deform $f$ to a new homeomorphism $g$ with the origin as the only fixed point in $B_{1}$. Since ind $(f, C) \neq 0$, the origin is an essential fixed point of $g$. This completes the proof that we can deform $f$ by an isotopy so that each arc component $C$ of Fix $(f)$ becomes a single point, or totally disappears, depending on whether $C$ is an essential fixed point class of $f$.

Suppose $x$ is an isolated inessential fixed point of $f$. Since $f$ is standard of on $N\left(\mathcal{T}^{\prime \prime}\right)$, this implies that $x$ is a flipped-pseudo-Anosov type fixed point in the interior of some $M_{i}$, so we can remove it using Lemma 5.7. If $C$ is a circle component of Fix $(f)$, we can remove it using Lemma 5.5. These, together with the above isotopies near each arc component of Fix $(f)$, will deform $f$ to a new homeomorphism $g$ such that each component of Fix $(g)$ is an essential fixed point class. Therefore, \#Fix $(g)=N(f)$.

Define an orbifold $X(M)$ to be small if it is a sphere with a total of at most three holes or cone points, or a projective plane with a total of 2 holes or cone points. Note that any homeomorphism on a small orbifold is isotopic to a periodic map.

Theorem 9.2. Let $M$ be a closed orientable 3-manifold which is either Haken or geometric. Then any orientation-preserving homeomorphism $f$ on $M$ is isotopic to a fixed point free homeomorphism, unless some component of the JSJ decomposition of $M$ is a Seifert fiber space with big orbifold. 
Proof. This follows from Proposition 7.5 if $M$ is a Sol manifold, and from Corollary 8.2 if $M$ is hyperbolic. If $M$ is a Seifert fiber space with $X(M)$ a sphere with at most 3 cone points, then by Theorem 3.11, $f$ is isotopic to a fiber-preserving homeomorphism. It follows immediately from Proposition 6.4 that $f$ is isotopic to a fixed point free map.

Now suppose $M$ has a nonempty JSJ decomposition. Let $\mathcal{T}$ be the decomposition tori, and deform $f$ by an isotopy so that $f(N(\mathcal{T}))=N(\mathcal{T})$. By the same argument as above, we may assume that the restriction of $f$ on each component $M_{i}$ of $M-\operatorname{Int} N(\mathcal{T})$ is a type 2 standard map.

Let $T$ be a component of $\mathcal{T}$, and assume that $f$ maps $N(T)$ to itself. Suppose $f$ exchange the two boundary components of $N(T)$. Then $f^{2}$ maps each piece $M_{i}$ adjacent to $N(T)$ to itself. Since $M_{i}$ is either hyperbolic or Seifert fibered, $\left.f^{2}\right|_{M_{i}}$ is isotopic to a periodic or Seifert fiber-preserving homeomorphism, so the restriction of $f^{2}$ to the boundary of $N(T)$ can not be isotopic to an Anosov map. By Lemma 7.2(1), $\left.f\right|_{N(T)}$ is rel $\partial N(T)$ isotopic to a fixed point free homeomorphism, which is automatically a type 2 map. If $f$ maps each boundary component of $N(T)$ to itself, then $f$ is already a type 2 standard map on the two pieces adjacent to $N(T)$, so by Lemma 7.2(2) $\left.f\right|_{N(T)}$ is also rel $\partial N(T)$ isotopic to a type 2 map.

After the above isotopy, we get a homeomorphism $f$ which is of type 2 on each $M_{i}$ and $N(T)$. Thus each component of Fix $(f)$ is a circle, which can be removed by Lemma 5.5 .

\section{References.}

[Be] L. Bers, An extremal problem for quasiconformal mapping and a theorem by Thurston, Acta Math., 141 (1978), 71-98.

[BO] M. Boileau and J. Otal, Scindements de Heegaard et groupe des homeotopies des petites varietes de Seifert, Invent. Math., 106 (1991), 85-107.

[BZ] M. Boileau and B. Zimmermann, The $\pi$-orbifold group of a link, Math. Z., 200 (1989), 187-208.

[B] F. Bonahon, Difféotopies des espaces lenticulaires, Topology, 22 (1983), 305-314.

[Br] R. Brown, The Lefschetz Fixed Point Theorem, Scott-Foresman, Chicago, 1971. 
[CB] A. Casson and S. Bleiler, Automorphisms of Surfaces after Nielsen and Thurston, London Math. Soc., Student Texts, 9 (1988).

[CV] L. Charlep and A. Vasquez, Compact flat Riemannian manifolds II I: the group of affinities, Amer. J. Math., 95 (1973), 471-494.

[FLP] A. Fathi, F. Laudenbach and V. Poenaru, Travaux de Thurston sur les surfases, Astérisque, 66-67 (1979).

[Ga] D. Gabai, On the geometric and topological rigidity of hyperbolic 3-manifolds, J. Amer. Math. Soc., 10 (1997), 37-74.

[GMT] D. Gabai, G.R. Meyerhoff, and N. Thurston, Homotopy Hyperbolic 3-Manifolds are Hyperbolic, MSRI Preprint \#1996-058.

[He] J. Hempel, 3-manifolds, Annals of Math. Studies 86, Princeton University Press, 1976.

[HR] C. Hodgson and J.H. Rubinstein, Involutions and isotopies of lens spaces, in 'Knot Theory and Manifolds', Lecture Notes in Math., 1144 (1983), 60-96.

[Iv] N.V. Ivanov, Nielsen numbers of self-maps of surfaces, J. Sov. Math., 26 (1984), 1636-1641.

[Ja] W. Jaco, Lectures on Three-Manifold Topology, in 'Regional Conference Series in Mathematics', American Math. Soc., 43 (1977).

[J1] B. Jiang, Lectures on Nielsen Fixed Point Theory, Contemp. Math., 14, Amer. Math. Soc., Providence, RI, 1983.

[J2] B. Jiang, Fixed points and braids, Invent. Math., 272 (1985), 249256.

[J3] B. Jiang, Fixed points of surface homeomorphisms, Bull. Amer. Math. Soc., 5 (1981), 176-178.

[JG] B. Jiang and J. Guo, Fixed points of surface diffeomorphisms, Pacific J. Math., 160 (1993), 67-89.

[JW] B. Jiang and S. Wang, Lefschetz numbers and Nielsen numbers for homeomorphisms on aspherical manifolds, in 'Topology Hawaii', edited by K.H. Dovermann, World Scientific Publishing Co., (1992), 119-136. 
[Ke] M. Kelly, The Nielsen number as an isotopy invariant, Topology Appl., 62 (1995), 127-143.

[Ki] T. Kiang, The theory of fixed point classes, Springer-Verlag, BerlinNew York, 1989.

[La] F. Laudenbach, Topologie de la dimension trois: homotopie et isotopie, Astérisque, 12 (1974).

[Li] W.B.R. Lickorish, Homeomorphisms of nonorientable two-manifolds, Math. Proc. Camb. Phil. Soc., 59 (1963), 307-317.

[Mo] J. Morgan, On Thurston's uniformization theorem for three-dimensional manifolds, in 'The Smith Conjecture', Academic Press, New York, (1984), 37-125.

[Or] P. Orlik, Seifert manifolds, Lecture Notes in Mathematics, 291 (1972).

[Ro] D. Rolfsen, Knots and Links, Publish or Perish, 1976.

[R] J.H. Rubinstein, On 3-manifolds that have finite fundamental groups and contain Kleinian bottles, Trans. Amer. Math. Soc., 351 (1979), 129-137.

[RB] J.H. Rubinstein and J. Birman, One-sided Heegaard splittings and homeotopy groups of some 3-manifolds, Proc. London Math. Soc., 49 (1984), 517-536.

[Sc] P. Scott, The geometry of 3-manifolds, Bull. London Math. Soc., 15 (1983), 401-487.

[Sc2] P. Scott, Homotopy implies isotopy for some Seifert fibre spaces, Topology, 24 (1985), 341-351.

[Th1] W. Thurston, The Geometry and Topology of 3-manifolds, Princeton University, 1978.

[Th2] W. Thurston, Three dimensional manifolds, Kleinian groups and hyperbolic geometry, Bull. Amer. Math. Soc., 6 (1982), 357-381.

[Th3] W. Thurston, On the geometry and dynamics of diffeomorphisms of surfaces, Bull. Amer. Math. Soc., 19 (1988), 417-431. 
[Wa] F. Waldhausen, On irreducible 3-manifolds which are sufficiently large, Annals of Math., 87 (1968), 56-88.

[Wa2] F. Waldhausen, Heegaard-Zerlegungen der 3-Sphäre, Topology, 7 (1968), 195-203.

[WW] S. Wang and Y-Q.Wu, Covering invariants and cohopficity of 3manifold groups, Proc. London Math. Soc., 68 (1994), 203-224.

[We] F. Wecken, Fixpunktklassen III, Math. Ann., 118 (1942), 544-577.

[Wu] Y-Q. Wu, Canonical reducing curves of surface homeomorphism, Acta Math. Sinica (New Series), 3 (1987), 305-313.

Department of Mathematics

PEKING UNIVERSITY

BEIJING 100871

China

E-mail address: jiangbj@sxx0.math.pku.edu.cn

Department of Mathematics

PEKING UNIVERSITY

BEIJING 100871

ChINA

E-mail address: swang@sxx0.math.pku.edu.cn

Department of Mathematics

UNIVERSITY OF IOWA

IowA CiTy, IA 52242

E-mail address: wu@math.uiowa.edu

ReCeived June 8, 1999. 